\title{
Understanding star formation in molecular clouds
}

\section{Effects of line-of-sight contamination on the column density structure ${ }^{\star}, \star$}

\author{
N. Schneider ${ }^{1,2}$, V. Ossenkopf ${ }^{3}$, T. Csengeri ${ }^{4}$, R. S. Klessen ${ }^{5,6,7}$, C. Federrath ${ }^{8,9}$, P. Tremblin $^{10,11}$, P. Girichidis ${ }^{12}$, \\ S. Bontemps ${ }^{1,2}$, and Ph. André ${ }^{13}$ \\ 1 Univ. Bordeaux, LAB, UMR 5804, 33270 Floirac, France \\ e-mail: nschneid@cea.fr \\ 2 CNRS, LAB, UMR 5804, 33270 Floirac, France \\ 3 I. Physikalisches Institut, Universität zu Köln, Zülpicher Straße 77, 50937 Köln, Germany \\ ${ }^{4}$ Max-Planck Institut für Radioastronomie, Auf dem Hügel 69, Bonn, Germany \\ 5 Universität Heidelberg, Zentrum für Astronomie, Institut für Theoretische Astrophysik, 69120 Heidelberg, Germany \\ ${ }^{6}$ Kavli Institute for Particle Astrophysics and Cosmology, Stanford University, SLAC National Accelerator Laboratory, \\ CA 94025, USA \\ 7 Department of Astronomy and Astrophysics, University of California, Santa Cruz, CA 95064, USA \\ 8 Monash Centre for Astrophysics, School of Mathematical Sciences, Monash University, VIC 3800, Australia \\ 9 Research School of Astronomy \& Astrophysics, The Australian National University, Canberra, ACT 2611, Australia \\ 10 Astrophysics Group, University of Exeter, EX4 4QL Exeter, UK \\ 11 Maison de la Simulation, CEA-CNRS-INRIA-UPS-UVSQ, USR 3441, CEA Saclay, France \\ 12 Max-Planck Institut für Astrophysik, 85741 Garching, Germany \\ 13 IRFU/SAp CEA/DSM, Laboratoire AIM CNRS - Université Paris Diderot, 91191 Gif-sur-Yvette, France
}

Received 4 February 2014 / Accepted 16 December 2014

\section{ABSTRACT}

\begin{abstract}
Column-density maps of molecular clouds are one of the most important observables in the context of molecular cloud- and starformation (SF) studies. With the Herschel satellite it is now possible to precisely determine the column density from dust emission, which is the best tracer of the bulk of material in molecular clouds. However, line-of-sight (LOS) contamination from fore- or background clouds can lead to overestimating the dust emission of molecular clouds, in particular for distant clouds. This implies values that are too high for column density and mass, which can potentially lead to an incorrect physical interpretation of the column density probability distribution function (PDF). In this paper, we use observations and simulations to demonstrate how LOS contamination affects the PDF. We apply a first-order approximation (removing a constant level) to the molecular clouds of Auriga and Maddalena (low-mass star-forming), and Carina and NGC 3603 (both high-mass SF regions). In perfect agreement with the simulations, we find that the PDFs become broader, the peak shifts to lower column densities, and the power-law tail of the PDF for higher column densities flattens after correction. All corrected PDFs have a lognormal part for low column densities with a peak at $A_{v} \sim 2$ mag, a deviation point (DP) from the lognormal at $A_{v}(\mathrm{DP}) \sim 4-5 \mathrm{mag}$, and a power-law tail for higher column densities. Assuming an equivalent spherical density distribution $\rho \propto r^{-\alpha}$, the slopes of the power-law tails correspond to $\alpha_{\mathrm{PDF}}=1.8,1.75$, and 2.5 for Auriga, Carina, and NGC 3603. These numbers agree within the uncertainties with the values of $\alpha \approx 1.5,1.8$, and 2.5 determined from the slope $\gamma$ (with $\alpha=1-\gamma$ ) obtained from the radial column density profiles $\left(N \propto r^{\gamma}\right)$. While $\alpha \sim 1.5-2$ is consistent with a structure dominated by collapse (local free-fall collapse of individual cores and clumps and global collapse), the higher value of $\alpha>2$ for NGC 3603 requires a physical process that leads to additional compression (e.g., expanding ionization fronts). From the small sample of our study, we find that clouds forming only low-mass stars and those also forming high-mass stars have slightly different values for their average column density $\left(1.8 \times 10^{21} \mathrm{~cm}^{-2}\right.$ vs. $\left.3.0 \times 10^{21} \mathrm{~cm}^{-2}\right)$, and they display differences in the overall column density structure. Massive clouds assemble more gas in smaller cloud volumes than low-mass SF ones. However, for both cloud types, the transition of the PDF from lognormal shape into power-law tail is found at the same column density (at $A_{v} \sim 4-5 \mathrm{mag}$ ). Low-mass and high-mass SF clouds then have the same low column density distribution, most likely dominated by supersonic turbulence. At higher column densities, collapse and external pressure can form the power-law tail. The relative importance of the two processes can vary between clouds and thus lead to the observed differences in PDF and column density structure.
\end{abstract}

Key words. dust, extinction - ISM: clouds - submillimeter: ISM - methods: data analysis - ISM: general

\section{Introduction}

Recent years have seen significant progress in understanding the link between the column density and spatial structure of molecular clouds and star formation. Molecular line surveys enabled

\footnotetext{
* Appendices are available in electronic form at http://www . aanda.org

$\star \star$ Herschel maps as FITS files are only available at the CDS via anonymous ftp to cdsarc.u-strasbg. fr (130.79.128.5) or via http://cdsarc.u-strasbg.fr/viz-bin/qcat?]/A+A/575/A79
}

us to study the velocity structure of clouds and yielded substantial results. Just to name a few, the very complex velocity structure of filamentary clouds was shown in a large Taurus survey (Goldsmith et al. 2008; Hacar et al. 2013), the influence of largescale convergent flows as a possible formation mechanism for molecular clouds was demonstrated by Motte et al. (2014), and the importance of global collapse of filaments for the formation of OB clusters was shown in Schneider et al. (2010). However, molecular tracers are restrained by their critical densities and optical depths effects, thus limiting their functionality to study 
Table 1. Molecular cloud parameters from Herschel data.

\begin{tabular}{|c|c|c|c|c|c|c|c|c|c|c|}
\hline Cloud & $\begin{array}{c}D \\
{[\mathrm{kpc}]} \\
(1)\end{array}$ & $\begin{array}{c}M \\
{\left[10^{4} M_{\odot}\right]} \\
(2)\end{array}$ & $\begin{array}{c}\Sigma \\
{\left[M_{\odot} \mathrm{pc}^{-2}\right]} \\
(3)\end{array}$ & $\begin{array}{c}\left\langle N\left(\mathrm{H}_{2}\right)\right\rangle \\
{\left[10^{21} \mathrm{~cm}^{-2}\right]} \\
(4)\end{array}$ & $\begin{array}{c}\Delta A_{v} \\
{[\mathrm{mag}]} \\
(5)\end{array}$ & $\begin{array}{c}A_{v, \mathrm{pk}} \\
{[\mathrm{mag}]} \\
(6)\end{array}$ & $\begin{array}{c}A_{v}(\mathrm{DP}) \\
{[\mathrm{mag}]} \\
(7)\end{array}$ & $\begin{array}{l}\sigma_{\eta} \\
(8)\end{array}$ & $\begin{array}{l}s \\
(9)\end{array}$ & $\begin{array}{l}\alpha \\
(10)\end{array}$ \\
\hline \multicolumn{11}{|c|}{ High-mass SF regions } \\
\hline NGC 3603 & 7.0 & $50.4(97.1)$ & $60(116)$ & $3.24(6.24)$ & $3.0 \pm 0.5$ & $1.6(4.8)$ & $4.9(8.1)$ & $0.52(0.27)$ & $-1.31(-1.61)$ & $2.53(2.24)$ \\
\hline Carina & 2.3 & $34.5(59.5)$ & $50(89)$ & $2.79(4.79)$ & $2.0 \pm 0.2$ & $2.6(4.4)$ & $5.5(7.4)$ & $0.38(0.20)$ & $-2.66(-3.04)$ & $1.75(1.66)$ \\
\hline \multicolumn{11}{|c|}{ Low-mass SF regions } \\
\hline Maddalena & 2.2 & $35.2(68.2)$ & $37(76)$ & $2.13(4.13)$ & $2.0 \pm 0.25$ & $1.9(3.9)$ & $4.9(7.8)$ & $0.32(0.20)$ & $-3.69(-5.21)$ & $1.54(1.38)$ \\
\hline Auriga & 0.45 & $2.2(3.7)$ & $28(47)$ & $1.51(2.52)$ & $0.8 \pm 0.1$ & $1.4(2.3)$ & $3.5(4.0)$ & $0.45(0.25)$ & $-2.54(-3.05)$ & $1.79(1.66)$ \\
\hline$\langle$ Corrected $\rangle$ & & & $44 \pm 7$ & $2.42 \pm 0.38$ & & $1.88 \pm 0.26$ & $4.70 \pm 0.42$ & $0.42 \pm 0.04$ & $-2.55 \pm 0.49$ & $1.90 \pm 0.22$ \\
\hline$\langle$ Original $\rangle$ & & & $82 \pm 14$ & $4.42 \pm 0.77$ & & $3.85 \pm 0.55$ & $6.83 \pm 0.95$ & $0.23 \pm 0.02$ & $-3.22 \pm 0.74$ & $1.74 \pm 0.18$ \\
\hline
\end{tabular}

Notes. All values in parenthesis are the ones determined from the original maps before correction for LOS contamination. The last two lines give the average values (and standard deviation) from the corrected and uncorrected maps. (1) Distance $D$ of the cloud. (2) Mass $M \propto N\left(\mathrm{H}_{2}\right) D^{2}$. The $\mathrm{H}_{2}$ column density determination assumes a mean atomic weight per molecule of 2.3. The total mass was determined above an $A_{v}$ level of $\sim 1 \mathrm{mag}$, which is a typical value for estimating molecular cloud extent (e.g., Lada et al. 2010). For the uncorrected maps, we determine the mass within the same area (above a threshold of $A_{v}=1+\Delta A_{v}[\mathrm{mag}]$ ). We note that for Auriga, the mass estimated by Harvey et al. (2013) is slightly higher $\left(4.9 \times 10^{4} M_{\odot}\right)$ than our value. Both mass values were derived from an area of $\sim 13 \mathrm{deg}^{2}$, while the mass of Auriga given in Lada et al. (2010) of $1.1 \times 10^{5} M_{\odot}$ is determined from the whole cloud complex, covering $80 \mathrm{deg}^{2}$. (3) Surface density $(\Sigma=\mathrm{M} /$ area). (4) Average column density (above a level of $10^{21} \mathrm{~cm}^{-2}$ ). (5) Background/foreground level of visual extinction. The error is the root mean square from the pixel statistics used to determine the contamination. (6) Peak of PDF in visual extinction. (7) Visual extinction value of the deviation point where the PDF starts to deviate from the lognormal shape at high column densities. (8) Dispersion of the fitted lognormal PDF. (9) Slope $s$ of the high-density tail of the PDF, determined by linear regression (the $\chi^{2}$ value is given in the panels of the PDF). We excluded the noisier and less well sampled points at the high column density end of the PDF. (10) Exponent of the spherical density distribution $\rho \propto r^{-\alpha}$, determined from $s$ with $\alpha=-2 / s+1$ (Federrath \& Klessen 2013).

only certain gas phases (for example, low- $J$ CO lines for lowdensity gas or $\mathrm{N}_{2} \mathrm{H}^{+}$for cold, high-density gas). On the other hand, widefield extinction maps obtained by near-IR colorexcess techniques cover a wider range of column densities, typically from $N\left(\mathrm{H}+\mathrm{H}_{2}\right) \sim 0.1-40 \times 10^{21} \mathrm{~cm}^{-2}$ (Lada et al. 1994; Lombardi \& Alves 2001; Lombardi 2009; Cambrésy et al. 2011, 2013; Rowles \& Froebrich 2009; Schneider et al. 2011). Some major results obtained from these studies are, for example, that there is a relation between the rate of star formation and the amount of dense gas in molecular clouds (Lada et al. 2010), that a universal threshold in visual extinction of $A_{v} \approx 6$ mag for the formation of stars could exist (Froebrich \& Rowles 2010), and that there are characteristic size scales in molecular clouds, indicating the dissipation and injection scales of turbulence (Schneider et al. 2011).

The large-scale far-infrared dust emission photometric observations of Herschel ${ }^{1}$ (Pilbratt et al. 2010) allow us now to make column density maps with a very wide dynamic range $N\left(\mathrm{H}_{2}\right) \sim 10^{20} \mathrm{~cm}^{-2}$ to a few hundred $10^{23} \mathrm{~cm}^{-2}$ at an angular resolution of $\sim 25^{\prime \prime}$ to $\sim 36^{\prime \prime}$ that provide an exceptional database to better understand the composition and structure of the interstellar medium. One result of Herschel is the importance of filaments for the star-formation process. Though the filamentary structure of molecular clouds has always been recognized, only the detailed investigation of the column density structure of filaments (Molinari et al. 2010; Arzoumanian et al. 2011; Palmeirim et al. 2013), their link to core formation (e.g., André et al. 2010, 2014; Polychroni et al. 2013; Könyves et al., in prep.), and their high mass input to form OB clusters (Schneider et al. 2012; Hennemann et al. 2012) emphasized their role for the formation of stars.

Despite this progress, there are still a number of important questions that are open. 1 . What is the relative importance of turbulence, gravity, magnetic fields, and radiative feedback for regulating the overall column density structure of molecular clouds? 2. Are there differences in the column density structure

\footnotetext{
1 Herschel is an ESA space observatory with science instruments provided by European-led Principal Investigator consortia and with important participation from NASA.
}

of clouds forming low-mass stars or high-mass stars? 3. Is there a universal (column) density threshold for the formation of selfgravitating prestellar cores? 4. Does the star formation efficiency (SFE) and star-formation rate (SFR) depend on the column density structure of molecular clouds?

In a series of papers using column density maps obtained with Herschel data and with near-IR extinction and the results of numerical simulations, we address these questions. This paper makes a start with a detailed study of the validity of column density maps and their probability distribution functions (PDFs) obtained from Herschel. We show that line-of-sight (LOS) confusion, i.e., emission from diffuse dust mixed with low-density gas as well as denser clouds in front or behind the bulk emission of the molecular cloud, leads to a significant overestimation in the column density maps. Apart from this observational approach, we quantify how the PDF properties change by using numerical simulations in which we add noise and foreground and/or background emission to an uncontaminated PDF. Using the corrected maps, we then study their PDFs and column density profiles to address the question of whether all molecular clouds have a similar column density structure.

\section{Line-of-sight confusion in continuum maps}

A notorious problem of continuum maps (extinction maps or column density maps derived from spectral energy distribution (SED) fitting using Herschel) is LOS confusion. This is particularly true in the Galactic plane and along spiral arms where dust emission not related to the cloud can significantly add, hence artifically increase, the derived column density for the cloud studied at a single distance. However, it is not easy to distinguish between the bulk emission of the cloud and background or foreground contribution from unrelated clouds if the distance information is missing. A simple approach we present in this paper is to determine a mean value for the contaminating column density by averaging pixels outside the molecular cloud close to the map borders. In the figures of column densities shown in the Appendix, this area is outlined as a white, dashed polygon. We find values between $\Delta A_{v} \sim 0.8$ and 3 (see Table 1), 
with a standard deviation of 0.1 to 0.5 , which are then subtracted as an offset from the original column density map. Such a constant offset subtraction is also intrinsically a part of the NICER/NICEST extinction mapping techniques (see Lombardi \& Alves 2001; Lombardi et al. 2008); however, major uncertainty in the background determination in our method comes from the placement of the field and not so much from the variation within. It can be that the "reference field" is still too close to the cloud itself and may contain contributions from the cloud. We exclude this effect for Carina and Maddalena by comparing to the near-IR extinction maps (Bontemps, priv. comm., see Schneider et al. 2011, for the method) of the large-scale environment of the clouds. For Auriga, we are also well outside of the cloud, based on the extinction map shown in Lada et al. (2009). It is only for NGC 3603 that such a selection bias could potentially be a problem.

We emphasize that our approach is simple and ignores possible gradients and more generally the spatial variation of the emission distribution of clouds that are located along the LOS. (More distant background clouds are expected to be smaller than foreground clouds that are assumed to be bigger in angular size.) This can lead to unrealistic features in the PDF (see Sect. 6). For simplicity, we only consider correcting for foreground/background contamination here by subtracting a constant value, which already provides significantly more reliable estimates of the true column density and masses.

An alternative method of estimating the contamination is to use the velocity information from atomic hydrogen or molecular lines and to translate the observed intensity into $\mathrm{H}_{2}+\mathrm{HI}$ column density. This has been done for Herschel studies of NGC 6334 (Russeil et al. 2013) and W3 (Rivera-Ingraham et al. 2013). However, using ${ }^{12} \mathrm{CO}$ or ${ }^{13} \mathrm{CO}$ is of very limited use for more distant and massive clouds because these tracers are only sensitive up to an $A_{v}$ of typically $10 \mathrm{mag}$. Probability distribution functions of CO (Goldsmith et al. 2008; Goodman et al. 2009) are clipped at higher column densities owing to a high optical depth. Only recently has a special method been developed to obtain an $\mathrm{H}_{2}$ map from ${ }^{13} \mathrm{CO}$ and $\mathrm{C}^{18} \mathrm{O}$ observations in $\mathrm{W} 43$ that suffers less from a cut-off at high column densities (Carlhoff et al. 2013). However, all methods are still subject to other uncertainties, such as the variable $\mathrm{CO} / \mathrm{H}_{2}$ conversion factor or the variation in excitation temperature $T_{\mathrm{ex}}$ (see Fig. 11 in Carlhoff et al. 2013 that shows how the PDF shifts by changing only a few Kelvin $T_{\text {ex }}$ ).

\section{Probability distribution functions (PDFs) of column density}

\subsection{PDFs in theory}

The PDF of column density $N$ is defined as the probability of finding gas within a range $[N, N+\mathrm{d} N]$. The column density $N$ can be replaced by the visual extinction $A_{v}{ }^{2}$. We define $\eta \equiv \ln (N /\langle N\rangle)$ as the natural logarithm of the column density $N$, divided by the mean column density $\langle N\rangle$, and the quantity $p_{\eta}(\eta)$ then corresponds to the PDF of $\eta$ with the normalization $\int_{-\infty}^{+\infty} p_{\eta} \mathrm{d} \eta=\int_{0}^{+\infty} p_{N} \mathrm{~d} N=1$.

Volume density PDFs are an important tool for characterizing the properties of the interstellar medium (e.g., Padoan et al. 1997; Vazquez-Semadeni \& Garcia 2001; Burkhart et al. 2013); models of SFR, in particular the stellar initial mass function (e.g., Hennebelle \& Chabrier 2008, 2009;

\footnotetext{
${ }^{2} N\left(\mathrm{H}_{2}\right)=A_{v} 0.94 \times 10^{21} \mathrm{~cm}^{-2} \mathrm{mag}^{-1}$ (Bohlin et al. 1978).
}

Padoan \& Nordlund 2002); and the SFR (Krumholz \& McKee 2005; Federrath \& Klessen 2012). However, the $3 \mathrm{D}$-density distribution in a molecular cloud cannot be directly observed; instead, only the 2D-projection, i.e. the column density field, is accessible. Numerical simulations show that the column density PDFs have a smaller width than the density PDFs and can show slightly different shapes in the high- and lowdensity regimes (Federrath et al. 2010), but they are statistically equivalent (Federrath \& Klessen 2013). Any particular, single column density projection can deviate from the volume density PDF, but the column and volume density PDFs show the same features if isotropy is assumed and/or if averaged over many realizations and/or different projections (Vazquez-Semadeni \& Garcia 2001; Fischera \& Dopita 2004; Federrath et al. 2010). A method using the column density power spectrum has been developed by Brunt et al. (2010) to approximate the 3D-PDF from a 2D-PDF.

Generally, the shape and dispersion of the PDF are determined by the density variations in a turbulent medium due to the contribution of compressive forcing, the velocity structure (expressed by the sonic Mach number), and magnetic pressure (e.g., Molina et al. 2012; Federrath \& Klessen 2012). From a theoretical point of view, the PDF of a molecular cloud shows a lognormal distribution if the cloud structure is governed by isothermal supersonic turbulence alone and all turbulence modes are sufficiently statistically sampled in the observed density structure. If there are statistical density fluctuations and/or intermittency due to locally compressive turbulence, some deviations from the lognormal shape at higher column densities can be observed as seen in the PDF of Polaris (Schneider et al. 2013). Significant deviations are predicted when parts of the gas undergo gravitational collapse and are thus governed by their selfgravity (Klessen 2000; Federrath et al. 2008; Kritsuk et al. 2011; Ward et al. 2014). Passot \& Vazquez-Semadeni (1998) showed that for nonisothermal gas, a power-law regime forms as well. Depending on the polytropic exponent $\gamma_{\mathrm{p}}$ in the pressure term $P \propto \rho^{\gamma_{\mathrm{p}}}$ with density $\rho$, a power-law tail forms in the case of $\gamma_{\mathrm{p}}<1$ for high densities and $\gamma_{\mathrm{p}}>1$ for low densities. Several authors (Ballesteros-Paredes et al. 2011; Cho \& Kim 2011; Ward et al. 2014) indicated that PDFs from hydrodynamic simulations including gravity change during the cloud evolution, and Federrath \& Klessen (2013) showed in their models how the slope of the high-density tail of the PDF flattens with increasing SFE. Recently, Girichidis et al. (2014) have demonstrated analytically that free-fall contraction of a single core or an ensemble of collapsing spheres forms a power-law tail.

\subsection{PDFs from observations}

To observationally determine PDFs, the most common method so far has been to employ column density maps obtained from near-IR extinction (see Sect. 1) and to derive a normalized distribution of pixels vs. column density in logarithmic bins. The observed PDFs show a lognormal distribution over a range of low column densities, typically between $A_{v} \sim 0.5$ to $\sim 4$. A more or less lognormal distribution was reported for clouds (Lupus, Coalsack: Kainulainen et al. 2009; Polaris: Schneider et al. 2013; Chamaeleon III: Alves de Oliveira et al. 2014) where none or only a few pre- or protostellar cores were detected. Herschel studies of several star-forming molecular clouds (Tremblin et al. 2014; Schneider et al. 2012, 2013) have shown that the lognormal part of the PDF broadens in the presence of external pressure (e.g., expanding $\mathrm{H}_{\mathrm{II}}$ regions) and that even a second peak indicating the compressed shell - can appear. Deviations from 
the lognormal shape in the form of a power-law tail are found for low-to-intermediate-mass star-forming regions (Lombardi et al. 2008), where it was proposed that the excess at high column densities is related either to star-formation activity (Kainulainen et al. 2009) or to the pressure due to different phases in the interstellar medium (Kainulainen et al. 2011). Gravity as the clearly dominating (over pressure and magnetic fields) process to form a power-law tail was suggested by Froebrich \& Rowles (2010) and Schneider et al. (2013).

We here determine PDFs from Herschel column density maps on a $14^{\prime \prime}$ grid (see Appendix B). The maps are on average large $\left(>1^{\circ}\right)$ and have a size of a few hundred ${ }^{2}$ pixels up to a few thousand ${ }^{2}$ pixels. We choose a binsize of 0.1 in $\eta$ that provides the best compromise between resolving small features in the PDF and avoiding low pixel statistics. In Appendix A, we show that binning in smaller or larger bins or varing the pixelsize and resolution does not change the PDF properties (width, peak, slope of power-law tail). To derive the characteristic properties of the PDF (width $\sigma_{\eta}$, peak $A_{v \text {,pk }}$, deviation from the lognormal shape $\left.A_{v}(\mathrm{DP})\right)$, we fit the lognormal function

$$
p_{\eta} \mathrm{d} \eta=\frac{1}{\sqrt{2 \pi \sigma_{\eta}^{2}}} \exp \left[-\frac{(\eta-\mu)^{2}}{2 \sigma_{\eta}^{2}}\right] \mathrm{d} \eta
$$

where $\sigma_{\eta}$ is the dispersion and $\mu$ the mean logarithmic column density. We do this systematically by performing several fits on a grid of parameters for $\eta$ and $\mu$ and then calculating the positive and negative residuals. Since the power-law tail is expected to cover higher $\eta$ values than the lognormal part, we select fits with the least negative residuals in the fitting procedure. We then determine the range of lognormality, when the difference between the model PDF (Eq. (1)), and the measured PDF $p_{\eta}$ is less than three times the statistical noise in $p_{\eta}$.

As shown by various authors (e.g., Kainulainen et al. 2009; Hill et al. 2011; Russeil et al. 2013; Schneider et al. 2012, 2013), a power-law tail for high column densities emerges for starforming regions. We perform a linear regression fit in order to determine the slope $s$ of the power-law tail. The values taken into account start at the deviation point (DP) where the lognormal PDF turns into a power-law distribution and stop where the power law is no longer well defined (at high column densities) because of the lower pixel statistics caused by resolution effects. In case the tail is only due to gravity, and if we assume spherical symmetry, the PDF slope $s$ of the power-law tail is related to the exponent $\alpha$ of the radial density profile (Federrath \& Klessen 2013) with

$\rho \propto r^{-\alpha}$

and

$\alpha=-2 / s+1$.

\section{Herschel column density maps}

For our study, we obtained the raw data from the Herschel archive for the following sources (see Table 1): AurigaCalifornia: $\operatorname{RA}(2000)=4^{\mathrm{h}} 21^{\mathrm{m}}, \operatorname{Dec}(2000)=37^{\circ} 35^{\prime} ; l=$ $162.3663^{\circ}, b=-8.7607^{\circ}$, OT1 PI: P. Harvey, see Harvey et al. (2013); Maddalena: $\operatorname{RA}(2000)=6^{\mathrm{h}} 48^{\mathrm{m}}, \operatorname{Dec}(2000)=$ $-3^{\circ} 39^{\prime} ; l=215.7908^{\circ}, b=-2.4273^{\circ}$, OT2 PI: J. Kauffmann; NGC 3603: RA(2000) $=11^{\mathrm{h}} 15^{\mathrm{m}}, \operatorname{Dec}(2000)=-61^{\circ} 15^{\prime} ; l=$ $291.6000^{\circ}, b=-0.5180^{\circ}$, Herschel Infrared GALactic plane survey (Hi-GAL), Molinari et al. (2010); Carina: RA(2000) = $10^{\mathrm{h}} 43^{\mathrm{m}}, \operatorname{Dec}(2000)=-59^{\circ} 24^{\prime} ; l=287.2328^{\circ}, b=-0.5009^{\circ}$,
OT1 PI: T. Preibisch, see Preibisch et al. (2012), Gaczkowski et al. (2012), Roccatagliata et al. (2013). These clouds were selected to cover different masses, sizes, and levels of SF. The Maddalena cloud is very massive but shows only a low level of SF. Auriga is less massive but forms low-mass stars, and Carina and NGC 3603 are high-mass SF regions with associated OB clusters. The process of data reduction and determination of column density maps at an angular resolution of $\sim 36^{\prime \prime}$ is explained in Appendix B where we also show the resulting column density maps and individual $250 \mu \mathrm{m}$ maps.

\section{Column density maps and PDFs}

Figure 1 shows the column density maps of the four clouds, corrected for contamination that can directly be compared with the original ones in the Appendix. (Both are plotted in the same column density range starting at zero.)

For a correct interpretation of the PDFs, the (non)completeness of the map needs to be considered. Our Herschel maps cover most of the molecular cloud material but do not extend to the very low column density outskirts of the cloud. For example, the extinction map of Auriga from Lada et al. (2009), covers $\sim 80 \mathrm{deg}^{2}$ including gas with $A_{v} \lesssim 1$, while our Herschel map comprises an area of $\sim 13 \mathrm{deg}^{2}$. The lowest continuous contour that is about complete in this map corresponds roughly to the completeness limit, i.e., $A_{v} \sim 1$ for the corrected Auriga map. The limit determined this way is $A_{v} \sim 0.9,1.0$, and 1.3 for Maddalena, NGC 3603, and Carina (all for the corrected maps), which also corresponds to the typical value taken for defining molecular cloud extent (e.g., Lada et al. 2010). For simplicity, we take $A_{v}=1$ for all clouds as the completeness limit, though a strict upper limit is probably $30 \%-50 \%$ higher. For the uncorrected column densities, the completeness level is then the value determined from the corrected maps plus the contamination level. However, the additional uncertainty of determining the back- and foreground contribution means that this level is more uncertain.

We emphasize that even if we are not fully complete for the lowest column densities, the higher statistics of these low column density pixels does not change the PDF shape significantly as we show in Appendix A. There, we plot the PDF of Auriga above different thresholds of column density, starting with the noise level of $A_{v}=0.2$. In summary, incompleteness/cropping can remove parts of the lognormal low-column density part of the PDF but it does not change the slope of the power-law tail. Cropping can indeed become an important concern when studying infrared dark clouds, because these can be embedded in a dense molecular envelope (see Schneider et al. 2014, for examples). If the PDF is only constructed from higher column density pixels, such a PDF underestimates the true, underlying PDF and only the power-law tail remains. On the other hand, intentionally selecting subfields in a map to construct PDFs can be useful for the physical interpretation. This was done in Hill et al. (2011); Schneider et al. (2012); Russeil et al. (2013); and Tremblin et al. (2014) to seperate the effects of turbulence, gravity, and compression by ionization on the column density structure of a cloud.

The LOS contamination correction significantly reduces (up to a factor $\sim 2$ ) the absolute values for average column density $\left\langle N\left(\mathrm{H}_{2}\right)\right\rangle$, mass, and surface density (Table 1$)$. The total mass of the cloud from the uncorrected maps was determined above an $A_{v}$-level of $\sim 1 \mathrm{mag}$, and for the uncorrected maps, we determined the mass within the same area (above a threshold of $\left.A_{v}=1+\Delta A_{v}[\mathrm{mag}]\right)$. The values for $\left\langle N\left(\mathrm{H}_{2}\right)\right\rangle$ range 
N. Schneider et al.: Understanding star formation in molecular clouds. I.
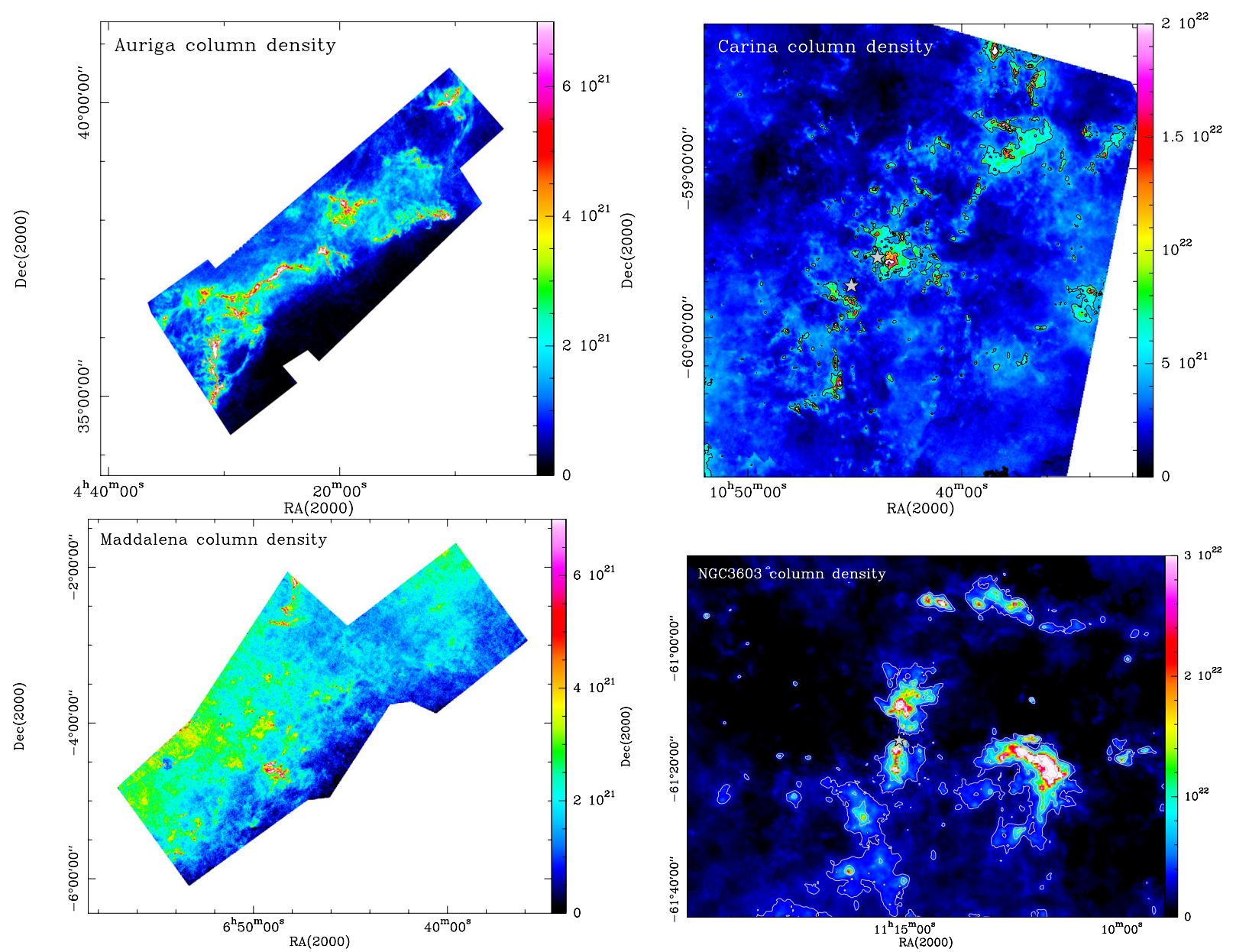

Fig. 1. Herschel column density maps (in $\left[\mathrm{cm}^{-2}\right]$, all starting at zero) of Auriga, Maddalena, NGC 3603, and Carina after correcting for line-ofsight contamination and removing noisy edges and areas where there was no overlap between PACS and SPIRE. The contour levels are 3, 6, 10, and $50 \times 10^{21} \mathrm{~cm}^{-2}$ for NGC 3606 and 5,10 , and $20 \times 10^{21} \mathrm{~cm}^{-2}$ for Carina.

between 1.5 and $3.2 \times 10^{21} \mathrm{~cm}^{-2}$ with an average value of $(2.4 \pm 0.4) \times 10^{21} \mathrm{~cm}^{-2}$. The surface density $\Sigma$ varies between 28 for Auriga and 60 for NGC 3603 with an average value of $\Sigma=44 M_{\odot} \mathrm{pc}^{-2}$ (instead of $82 M_{\odot} \mathrm{pc}^{-2}$ that was obtained from the original maps) $)^{3}$. This value corresponds very well to the one found by Heyer et al. (2009) with $\Sigma=42 M_{\odot} \mathrm{pc}^{-2}$ from a sample of clouds ( $>250$ ) from the FCRAO Galactic Ring Survey investigated with ${ }^{13} \mathrm{CO} 1 \rightarrow 0$ emission, and to the value $\Sigma=41 M_{\odot} \mathrm{pc}^{-2}$ obtained from extinction maps of five nearby clouds (Lombardi et al. 2010). However, we note that our simple approach may still under- or overestimate the LOS contamination. In a forthcoming paper (Paper III), we will present a study of more than 20 clouds to reach greater statistical significance.

The PDFs determined from the original and contamination corrected maps are shown in Figs. 2 and 3. The low column density regime of the uncorrected maps is limited by noise and LOS contamination of the map $\left(\triangle A_{v}\right.$-value listed in Table 1$)$, the vertical dashed line in Figs. 2 and 3 indicates the approximate completeness level (see above). For Auriga, the pixels left of the completeness limit still sample low-column density molecular cloud material, though we most likely miss the larger extent of this cloud component. We then fitted with a lognormal function

\footnotetext{
3 We use the threshold $A_{v}=1$ mag to "define" a molecular cloud. Taking a higher value such as $A_{v}=2 \mathrm{mag}$, we obtain a variation in $\Sigma$ between 47 and $90 M_{\odot} \mathrm{pc}^{-2}$ with an average value of $63 M_{\odot} \mathrm{pc}^{-2}$.
}

the lower extinction part of the PDF as explained in Sect. 3.14. For the LOS-corrected maps, the low extinction values (left of the PDF peak) do not neccessarily have a perfect lognormal distribution (e.g., Maddalena) because removing a constant offset can lead to negative pixels in the maps that are ignored during the process to make the PDF.

As outlined in Sect. 6, noise and "overcorrection" can lead to a Gaussian pixel distribution that shows up as a linear run in the low column density range of the PDF. We therefore slightly iterated the correction value for the contamination value in order to avoid this effect and optimized the threshold where we start the lognormal fit. The original Auriga PDF (see also Harvey et al. 2013) shows a superposition of two PDFs where the first (low extinction) peak is compatible with the contamination level of the map and nearly disappears when a level of $\Delta A_{v}=0.8$ is removed. In the case of Auriga, the correction works very well because the contamination is most likely a rather homogeneous layer in front of or behind the Auriga cloud. Such a superposition has already been observed in Pipe (Lombardi et al. 2006).

The LOS contamination correction has several effects: (i) the PDF is broader (increase of $\left\langle\sigma_{\eta}\right\rangle$ from $0.23 \pm 0.02$ to $0.42 \pm$ $0.04)$; (ii) the slope becomes flatter; and (iii) the peak and deviation point of the PDF from lognormal to excess $\left(A_{v}(\mathrm{DP})\right.$ from

4 We keep the classical approach to fit one lognormal PDF to the low column density range though other functional fits, such as a Gaussian, can be possible as well (Alves et al. 2014). 

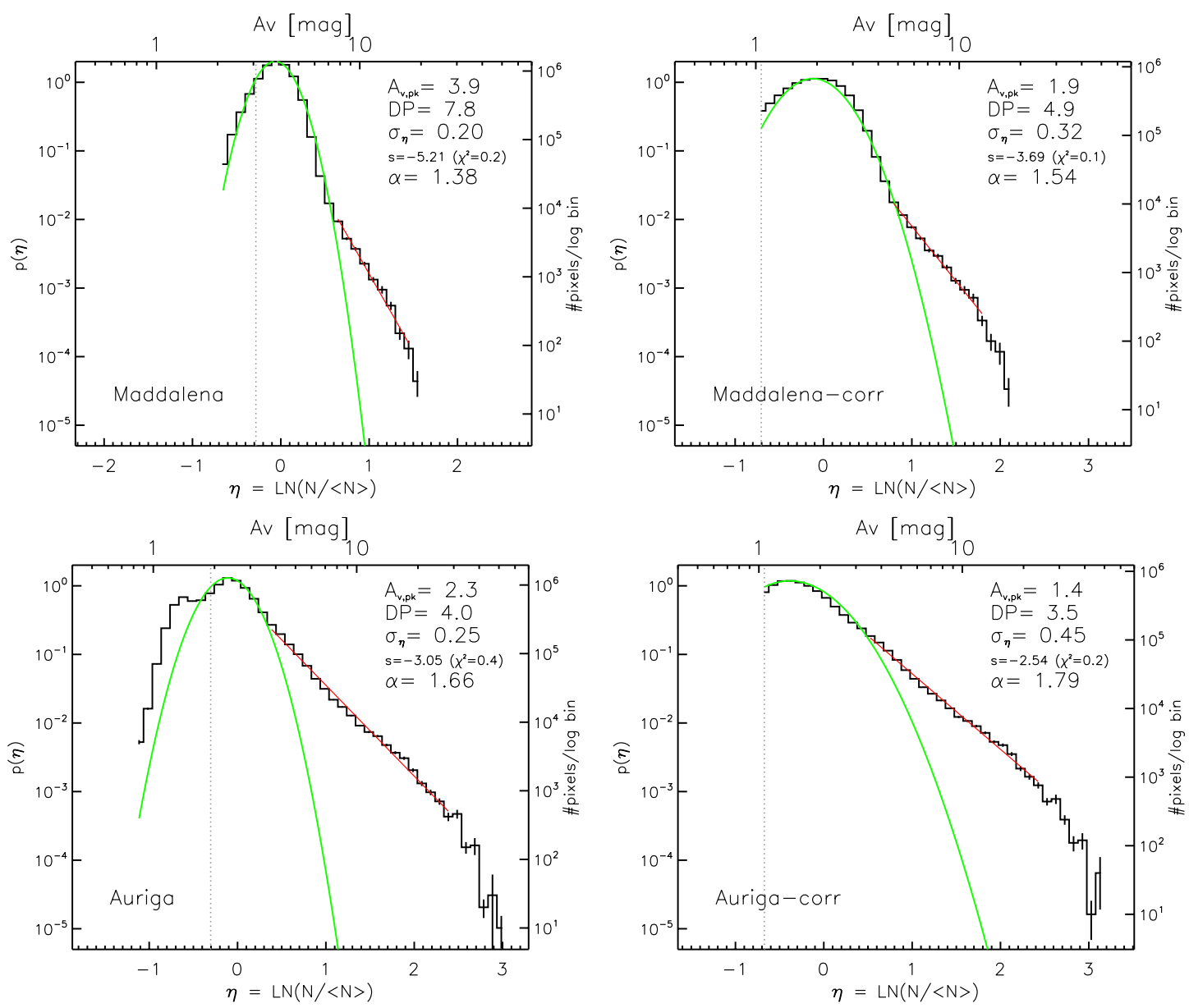

Fig. 2. PDFs derived from Herschel column density maps toward the low-mass star-forming regions Maddalena and Auriga. Left panel: PDF from the original map, right: one from the corrected map. The vertical dashed line indicates the approximate completeness limit. The left $y$-axis gives the normalized probability $p(\eta)$, the right $y$-axis the number of pixels per log bin. The upper $x$-axis is the visual extinction and the lower $x$-axis the logarithm of the normalized column density. The green curve indicates the fitted PDF. For Auriga, diffuse LOS-contamination (or a well defined seperate cloud) shows up as an individual PDF at low extinctions. The red line indicates a power-law fit to the high $A_{v}$ tail. Inside each panel, we give the value where the PDF peaks $\left(A_{v, \mathrm{pk}}\right)$, the deviation point from lognormal to power-law tail (DP), the dispersion of the fitted PDF $\left(\sigma_{\eta}\right)$, the slope $s$ and the $X^{2}$ of the fit (linear regression), and the exponent $\alpha$ of an equivalent spherical density distribution. These values are also summarized in Table 1.

now on) shift to lower values. In Sect. 6, we quantify these effects by an analytic model. The most dramatic change is observed for the PDFs of high-mass SF regions (Fig. 3). The very narrow distribution ${ }^{5}\left(\sigma_{\eta}=0.27\right)$ for NGC 3603 becomes much broader $\left(\sigma_{\eta}=0.52\right)$, the PDF peak shifts from $A_{v}=4.8$ mag to $1.6 \mathrm{mag}$, and $A_{v}$ (DP) from $8.1 \mathrm{mag}$ to $4.9 \mathrm{mag}$. The same applies for the Carina PDF with a change of $\sigma_{\eta}=0.2$ to 0.38 after correction $^{6}$. Other Herschel studies of high-mass SF regions, such as NGC 6334 (Russeil et al. 2013) where a LOS contamination of $\Delta A_{v} \sim 2-3$ mag was estimated, show the same narrow PDFs. It is thus essential to consider the contamination for a correct interpretation of PDFs for high-mass SF regions.

In Table 1 the PDF properties are listed, and they reveal that the correction leads to an equalization of the values for all

\footnotetext{
5 A low angular resolution (such as for extinction maps at a few arcmin resolution) also naturally results in narrower PDFs since the highest density structures are not resolved well. This effect can become important for very distant clouds.

6 Our PDF is different from the one shown in Preibisch et al. (2012) because their column density map was obtained from a fit using only the Herschel wavelengths 70 and $160 \mu \mathrm{m}$, which are not good tracers of cold gas.
}

clouds. The PDF peak values now have a range of 1.4 mag to $2.6 \mathrm{mag}$ instead of $2.3 \mathrm{mag}$ to $4.8 \mathrm{mag}$, and $A_{v}(\mathrm{DP})$ changes to $3.5 \mathrm{mag}$ to $5.5 \mathrm{mag}$ with an average of $4.7 \pm 0.4 \mathrm{mag}$. (Original values are $4.0 \mathrm{mag}$ to $8.1 \mathrm{mag}$ with an average of $6.8 \pm 1.0 \mathrm{mag}$.) A value of $A_{v}(\mathrm{DP})$ between 4 and 5 is less than what was found in other studies using extinction maps $\left(A_{v}(\mathrm{DP})=6\right.$ in Froebrich \& Rowles 2010), but consistent with recent hydrodynamic models of turbulent and self-gravitating gas (Ward et al. 2014).

\section{Simulations}

\subsection{Method}

We modeled the effect of LOS contamination in a numerical simulation, providing "ideal" PDFs consisting of a lognormal part and a power-law tail. Maps were generated with $500 \times 500$ pixels matching the typical observational map size and grid investigated in Sect. 5. To simulate maps that combine the given probability distribution of pixel values with spatial correlations among the pixels that are characteristic of observed maps (needed in Sect. 6.3), we started from fractional Brownian motion ( $\mathrm{fBm}$ ) fractal maps (Stutzki et al. 1998) with a power spectral index 
N. Schneider et al.: Understanding star formation in molecular clouds. I.
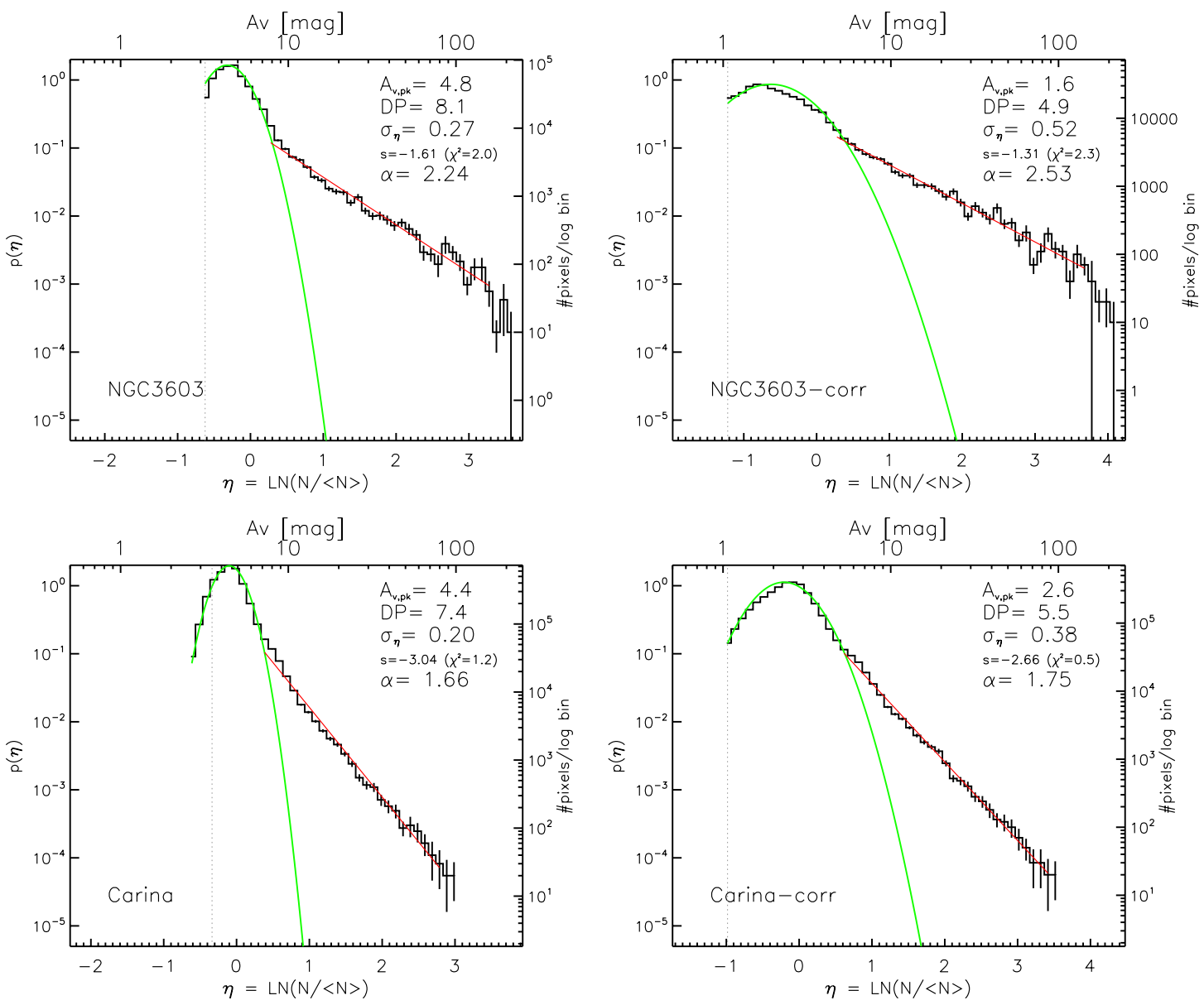

Fig. 3. PDFs derived from Herschel column density maps toward the high-mass star-forming regions NGC 3603 and Carina. Left panel: the PDF from the original map, right: one from the corrected map. All other parameters as in Fig. 1.

of 2.8, matching the typically observed spatial scaling behavior. Because the fBm maps are characterized by a normal distribution of values, we obtained the desired PDF in a second step by shifting each density by the value given by the ratio of the inverse integrals of the original and the desired PDFs. The result is visualized in Fig. 4. Larger and smaller fields were tested to verify the numerical accuracy of the method.

The generated field was then "contaminated" by adding a constant level to all map values and finally "observed" including Gaussian white noise typical of the Herschel observations. The overall model is characterized by six parameters: the width and the center $A_{v}$ of the lognormal PDF contribution, the exponent of the power-law tail, the deviation point characterizing the transition from the lognormal to the power-law PDF, the additive contamination $\Delta A_{v}$ and the standard deviation of the observational noise. To analyze the impact of the contamination we fixed all other parameters to lie within a range obtained for the observations in Sect. 5, i.e., a center (peak) at $A_{v}=2.0 \mathrm{mag}, \sigma_{\eta}=0.5$, $s=-2.0, A_{v}(\mathrm{DP})=4.3 \mathrm{mag}$, and a noise $\mathrm{rms} \sigma_{A_{V}}=0.1$.

\subsection{Effect of LOS-contamination on PDF properties}

In Fig. 5 it becomes obvious that the fit of the resulting measurable PDFs provides parameters that clearly deviate from the original input, in particular for the $\Delta A_{v}=3.0$ case. One observes that the lognormal part of the PDF is strongly compressed, consistent with what we observe for the NGC 3603 cloud (Fig. 3). The statistical sampling of that part becomes very rough. The value for $A_{v}(\mathrm{DP})$ increases, and the slope of the power-law tail

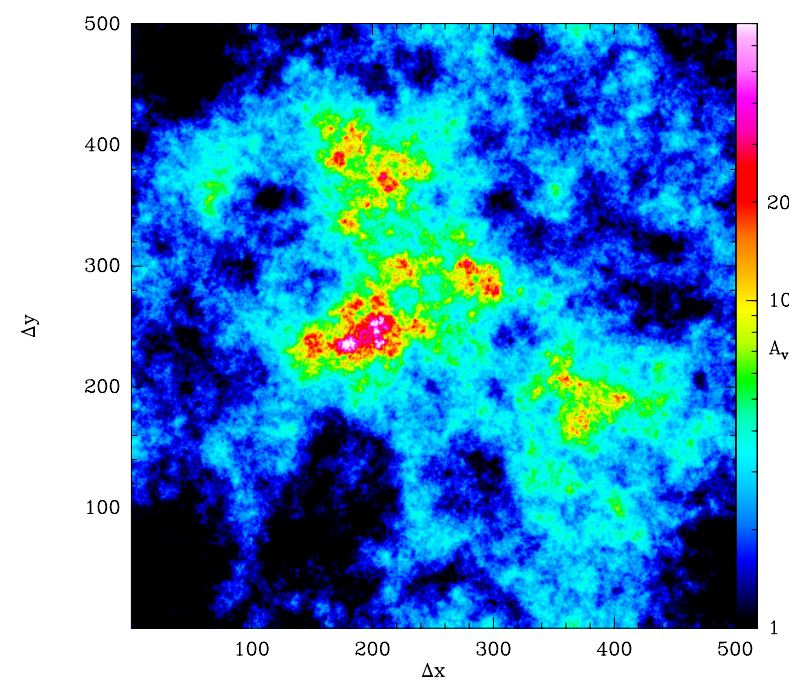

Fig. 4. Simulated map $(500 \times 500$ pixels $)$ characterized by a PDF with lognormal part and power-law tail, derived from a fractional Brownian motion (fBm) map with a power spectral index of 2.8. The column densities are expressed in $A_{v}$.

becomes steeper than $s=-2.0$ that characterized the original cloud $^{7}$.

7 The offset correction mathematically results in a modified function without a power-law tail. However, the corrections are small enough, so that fitting a power-law function and inferring a slope from this fitting function is still a reasonable procedure. 

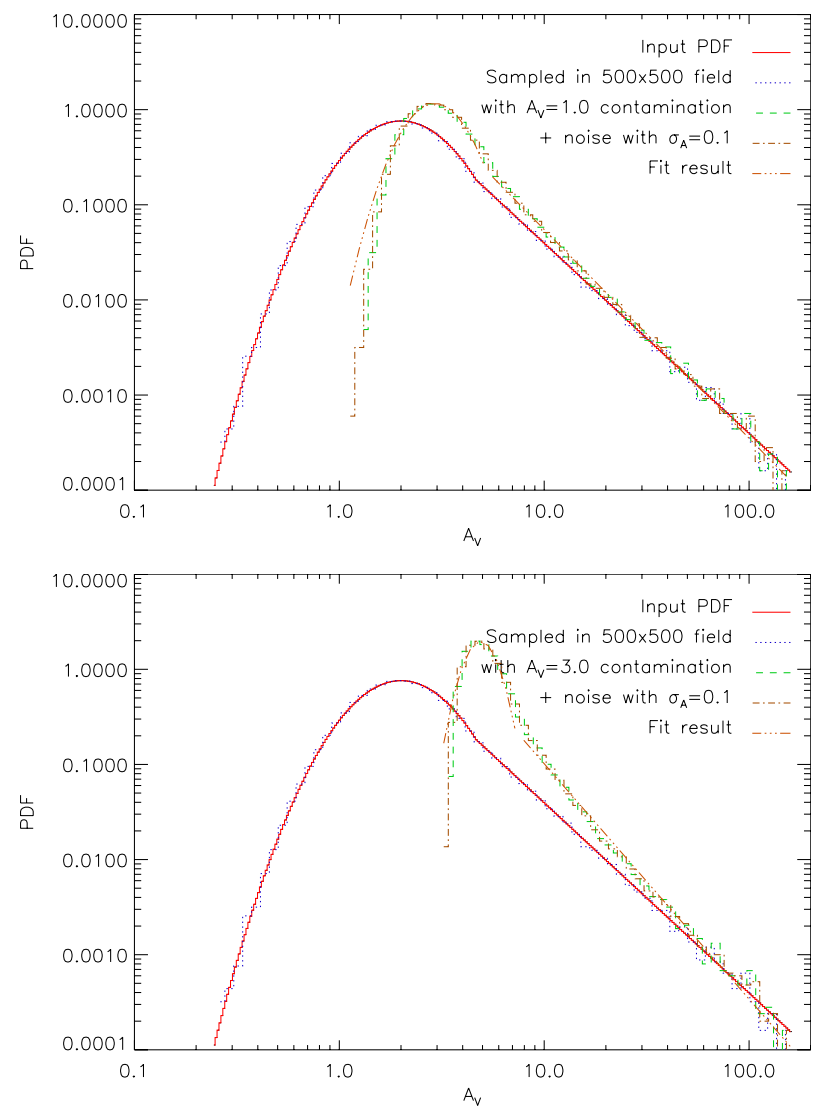

Fig. 5. Simulations of the PDF of an observed cloud with an originally perfectly lognormal core and a power-law tail (solid line). The representation by a finite-size map introduces some uncertainties (dotted line). The addition of an $\Delta A_{v}=1.0$ (3) contamination distorts the shape of the PDF and observational noise adds a low-exctinction component. The deviation point $A_{v}(\mathrm{DP})$ shifts by $\sim 4$ mag for a contamination of $\Delta A_{v}=1.0$ (3). The fit of the resulting PDF by a lognormal core and a power-law tail does not recover the initial parameters.

To quantify this effect systematically, we show in Fig. 6 the change in the width of the PDF and the slope of the power-law tail when measured as a function of LOS contamination, always starting from the standard parameters of the underlying cloud with $\sigma_{\eta}=0.5$ and $s=-2.0$. The small irregularities in the curve result from the discrete binning of the randomly sampled PDF. We find a dramatic effect in both parameters. For a contamination of $\Delta A_{v}=2, \sigma_{\eta}$ is already reduced by more than a factor two, and the slope of the power-law tail has steepened from -2.0 to -2.4 . For an $\Delta A_{v} \approx 10$, characterizing distant massive regions or infrared dark clouds, $\sigma_{\eta}$ has decreased by more than a factor of five and the power-law tail has a slope of -3.4 .

\subsection{Effect of finite resolution on the PDF}

Figure 7 shows the impact of a finite angular resolution on the PDF shape, which is implemented here as a convolution with a Gaussian beam of varying FWHM. We find the impact of a reduced resolution at very low column densities and at column densities above $A_{v}=15$. When reducing the resolution, the measured peak column densities drop. However, one clearly sees that a finite resolution only detracts from the highest peaks in the high-density PDF tail, but it does not change any of the properties of the PDF (peak, width, deviation point, slope) that we discuss here. However, lower resolution can lead to bumps and distortions in the general power-law tail. In real maps with
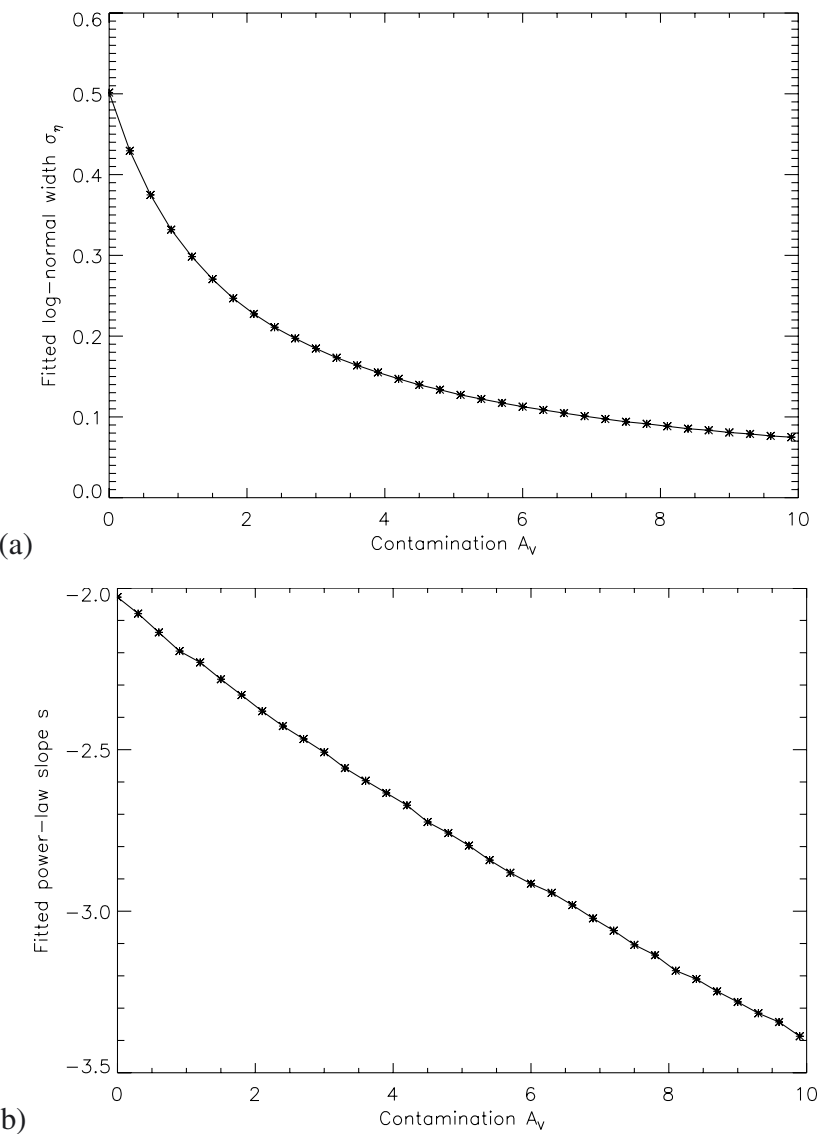

Fig. 6. Dependence of the fitted width of the lognormal core of the PDF a) and the fitted slope of the power-law tail b) as a function of the foreground (and/or background) contamination for the standard parameters of the molecular cloud PDFs.

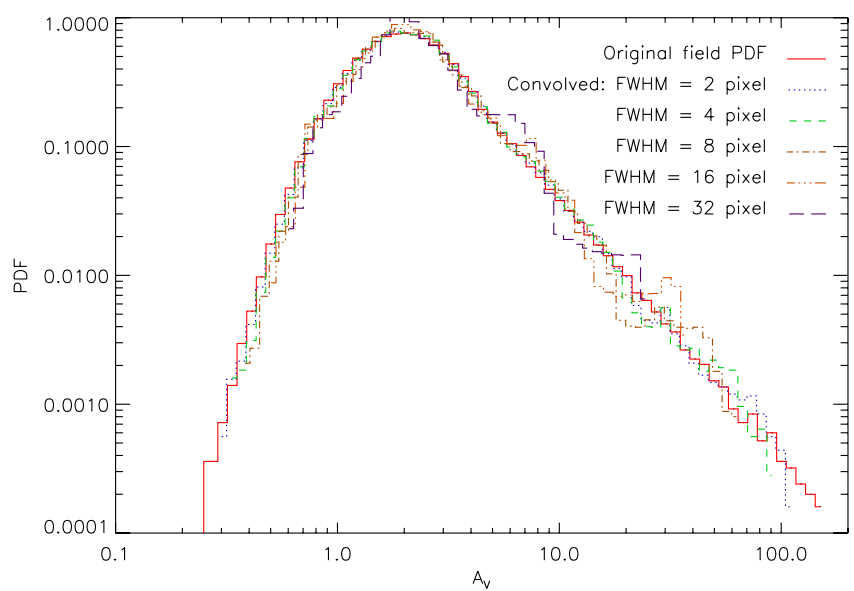

Fig. 7. Influence of finite beam size on the PDF shape. Different beam size (expressed as pixel size of $2,4,8,16$, and 32) were chosen. The convolution has a minor effect for the high-column density tail of the $\mathrm{PDF}$ and no influence on the lower-density lognormal distribution.

observational noise, the change at low densities would not be visible because the low column density part is more strongly affected by noise.

\subsection{Effect of noise and uncertainty on the correction}

The addition of observational noise adds a contribution to the structure that is hardly visible in the contaminated PDF, but that 
N. Schneider et al.: Understanding star formation in molecular clouds. I.

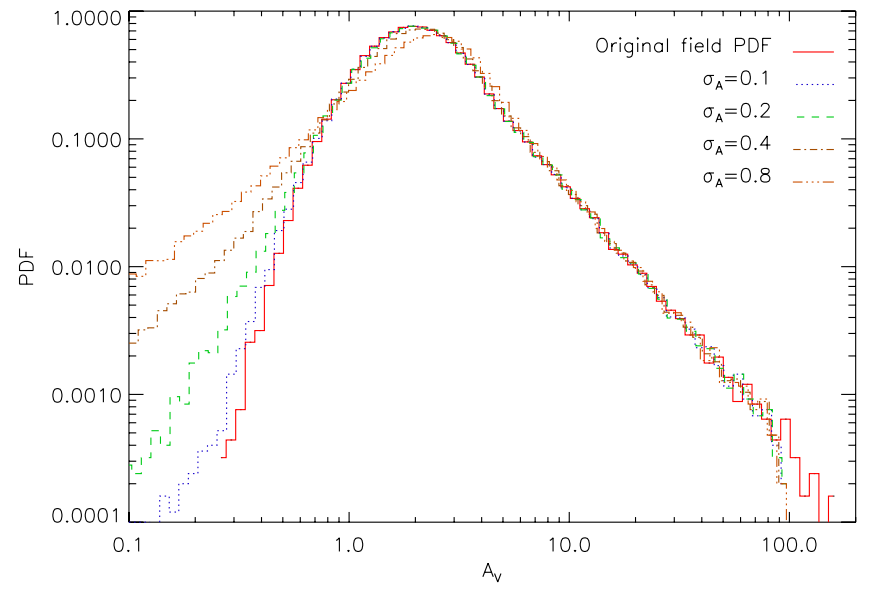

Fig. 8. Simulations of reconstructed PDFs observed with different amplitudes of noise $\sigma_{A}$, expressed in terms of visual extinction. The reconstruction does not depend on the absolute value of the foreground/background contamination.

may reappear as amplified after the correction of the contamination. The noise is assumed to have an approximately Gaussian distribution providing a floor of small fluctuations with zero average. In the logarithmic binning, the core of this Gaussian gives a linear contribution to the PDF for low densities, i.e. below the lognormal part of the PDF of the observed structure. This effect is simulated in Fig. 8 where we varied the observational noise from Fig. 5 and applied the contamination correction to reconstruct the PDF of the original structure.

We find a clear excess at low column densities, which turns close to the expected linear behavior for $\sigma_{A_{V}}=0.8$. Starting from $\sigma_{A_{V}}=0.4$, we also find a slight, but noticeable, shift in the PDF peak to higher $A_{v}$. For actually observed data, this should be taken into account. The effect is independent of the contamination that is added and subtracted in the PDF transformation.

The observed PDFs in Sect. 5 show the same kind of lowcolumn density excess as these simulations; in fact, the example of the Maddalena cloud matches the simulation for $\sigma_{A_{V}}=0.4$ exactly. However, the pure observational noise in the column density maps is much lower. This can be explained by additional small scale uncertainties and fluctuations in $\sigma_{A}$. They behave similarly to noise, but are not observational noise. They may represent fluctuations in the overall cloud contamination, either variations in the foreground screen or small background contaminating clouds (see also discussion in Alves et al. (2014) for correlated pattern of noise in extinction maps). As long as the fluctuations are relatively small, their impact on the fit of the main lognormal part of the PDF is negligible.

Very low column densities, giving rise to a linear contribution to the PDF for low $A_{v}$, can also stem from an "overcorrection" of the contamination, however, shifting part of the real cloud structure to column densities around zero. Therefore, using too high a value for the contamination correction by $\Delta A_{v}$, leads to a similar effect as increased noise. Figure 9 shows this for an example calculated with negligible noise $\left(\sigma_{A}=0.01\right)$ for different levels of $\Delta A_{v}$. We find a similar linear distribution for the low column-density pixels that becomes more important for increasing $\Delta A_{v}$. In this case, the peak of the PDF shifts in the other direction, compared to the addition of noise/fluctuations, i.e., to lower column densities.

When evaluating the low-column-density excess, we find that an overcorrection always has a stronger impact than noise or fluctuations of the same amplitude. Therefore we can analyze

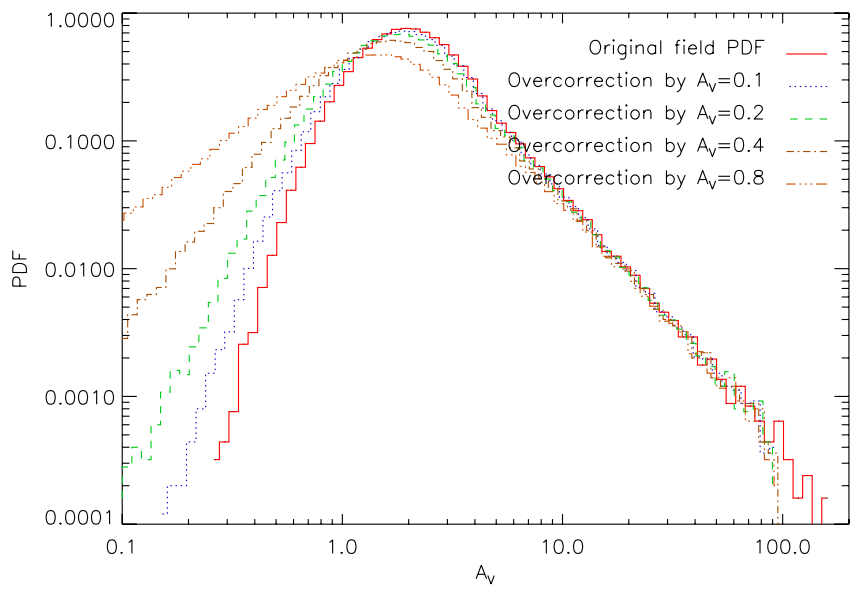

Fig. 9. Simulations of a PDF with negligible noise $\left(\sigma_{A}=0.01\right)$ when applying values that are too high for the contamination correction $\Delta A_{v}$. The reconstruction does not depend on the absolute value of the foreground/background contamination but only on the difference between actual contamination and subtracted contamination.

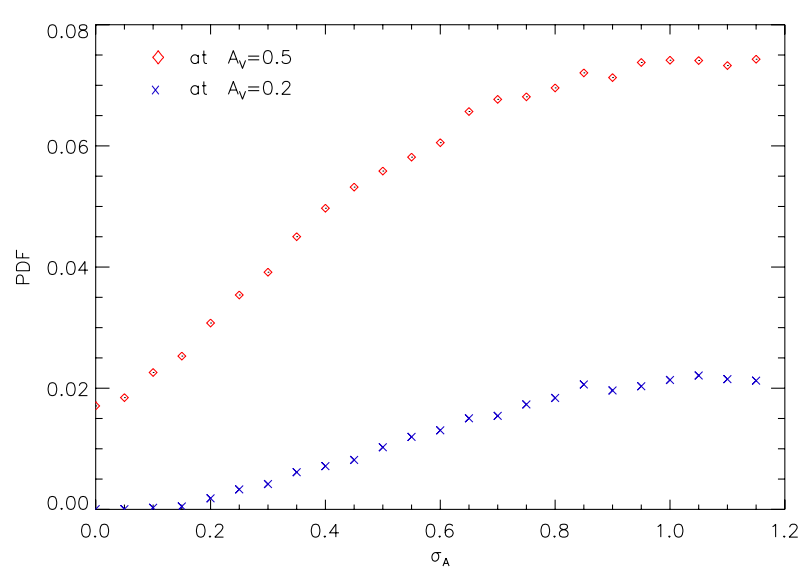

Fig. 10. Amplitude of the low column density in the PDF as a function of the added level of noise or small-scale fluctuations, corresponding to Fig. 8 for two points in the low-column density wing. The scatter in the points results from the adaptive binning in the PDF computation.

the low-density excess in the observations to get an upper limit of the uncertainty in the contamination correction when comparing the excess with the noise impact. This uncertainty then represents either fluctuations in the contamination or an absolute error in the contamination correction (in which case it would be somewhat overestimated). This is done through the simulation shown in Fig. 10. It gives the amplitude of the PDF at $A_{V}=0.2$ and $A_{V}=0.5$ as a function of the amplitude of the fluctuations; i.e., this quantifies the low-density excess in Fig. 8 for all possible noise/fluctuation levels. For all observations, we used this approach to quantify the uncertainty of the contamination correction, then excluding the low-density part from the fit of the lognormal part of the PDF of the actual cloud structure (see Sect. 5). Figure 11 illustrates an example of this procedure where we chose the "worst case" scenario for Maddalena. The PDF is plotted over a wide column density range, and the $A_{v}=0.5 \mathrm{mag}$ level corresponds to an amplitude of the PDF of $\approx 0.06$. Using Fig. 7 as a look-up table indicates that for this amplitude, the uncertainty of the correction corresponds to approximately $0.5 \mathrm{mag}$.

In this way, we not only can estimate the contamination of the cloud extinction by foreground or background material, but also the uncertainty of the contamination. The uncertainty can be 


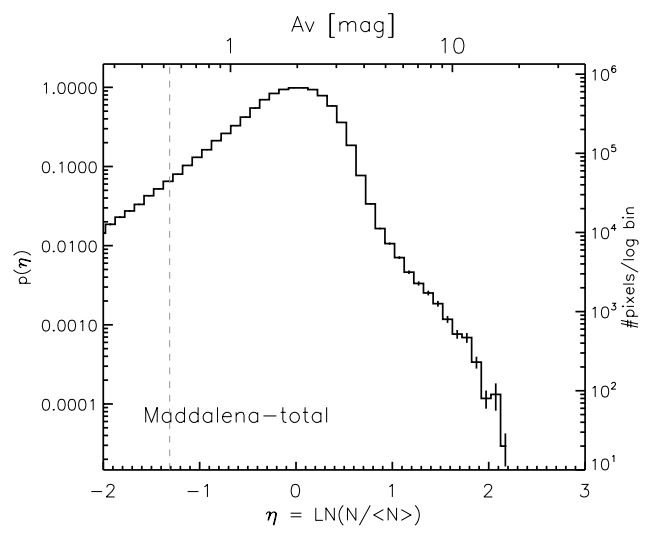

Fig. 11. PDF of Maddalena including the very low column density range down to $A_{v} \sim 0.2 \mathrm{mag}$. The dashed line indicates the $A_{v}=0.5 \mathrm{mag}$ level used to derive the amplitude of the PDF (in this case $\approx 0.06$ ) to be compared to Fig. 10 in order to determine the uncertainty of the correction (in this case $\approx 0.5-0.55$ ).

due to random fluctuations or a systematic error. For any measured low-density excess, the systematic error is always smaller than the random error, so that we provide an upper limit to the total uncertainty by giving only the magnitude of the possible fluctuations.

Summarizing, these findings clearly demand a correction of the contamination when analyzing observed column-density or extinction maps. As a first easy approach, the contamination can be removed from the maps before the analysis, or Fig. 6 can be used as a lookup table to obtain the original parameters from the measured width and power-law slope. In the next section, we discuss the physical implications (mean column density, column density structure, and cumulative mass function) of the corrected Herschel column density maps.

\section{Discussion}

\subsection{Do all molecular clouds have the same mean column density?}

The third Larson law (Larson 1981) is an empirical relation between the volume density $\rho$ of a cloud and its size $r$ with $\rho \propto r^{-1.1}$, and this implies that all molecular clouds have approximately constant mean column densities. However, there is a long-lasting controversy about its validity in theory (e.g., Ballesteros-Paredes \& Mac Low 2002) and observations (e.g., Schneider \& Brooks 2004; Heyer et al. 2009). Recently, a study of Lombardi et al. (2010), using extinction maps of five molecular clouds, has concluded that all clouds in their sample have identical average column densities above a given extinction threshold. However, their investigation does not include very massive and dense clouds. (The only high-mass SF region in their sample is Orion A with a column density below $10^{23} \mathrm{~cm}^{-2}$, see, e.g. Polychroni et al. 2013.)

We show that a correction of column density maps for the effect of LOS contamination leads to a typical value of $1.5-3 \times 10^{21} \mathrm{~cm}^{-2}$ for the average column density for the individual clouds with a total average for all clouds of $\left\langle N\left(\mathrm{H}_{2}\right)\right\rangle=$ $(2.4 \pm 0.4) \times 10^{21} \mathrm{~cm}^{-2}$. This range is fairly narrow, taking the whole dynamic range of column densities of molecular clouds into account across two magnitudes (a few $10^{21} \mathrm{~cm}^{-2}$ up to a few $10^{23} \mathrm{~cm}^{-2}$ ). There is a tendency for the high-mass SF clouds in our sample, NGC 3603 and Carina, to have slightly higher average column densities and surface densities compared to the low-mass SF clouds Auriga and Maddalena, but this difference in a sample of only four clouds is not yet significant and needs further studies. In any case, similar mean column densities for all cloud types do not imply that the global column density structure of all molecular cloud types is the same, as we discuss in the next section.

\subsection{Radial column density profiles}

Though the average column density in various cloud types shows no strong distinction, there is still a small difference between the low-mass SF regions Maddalena and Auriga $(\langle N\rangle=1.8 \times$ $\left.10^{21} \mathrm{~cm}^{-2}\right)$ and the high-mass SF regions Carina and NGC 3603 $\left(\langle N\rangle=3.0 \times 10^{21} \mathrm{~cm}^{-2}\right)$. Is this variation possibly because the clouds have a different column density structure across the cloud, with a common low-density regime but accumulation of denser gas in smaller volumes?

To test this, we determined radial column density profiles for three of our clouds in the sample. Figure 12 shows NGC 3603 and Carina, which are centrally condensed enough to enable such a study, while the Auriga cloud is very filamentary so that we selected only one prominent clump (in the center of the filament, see Fig. 1) with a somewhat circular shape. We excluded Maddalena because there is no such clump. We then determined the column density at each point on the maps depending on the distance to the center and plotted the resulting profiles in Fig. 13. The clouds are "cropped" because we need to focus on these spherical central regions.

At first glance, all profiles show no clear jump in column density at a certain threshold level in column density or radius. Because all clouds have significant substructure, some denser clumps and filaments protrude as peaks in the column density profile (e.g., at $6 \mathrm{pc}$ for Carina). Ignoring these peaks, the profiles show a rather smooth slope, though there is a tendency to a shallower slope for Carina and NGC 3603 toward the cloud center, i.e. lower radii $r$. The profiles were not deconvolved from the beam so that the result for NGC 3603 (resolution of $0.47 \mathrm{pc}$ ) is less reliable. Tremblin et al. (2014) and Didelon et al. (in prep.) have observed such a flattening of the slope toward the cloud center in M16 and Mon R2, which are both regions with closely associated $\mathrm{H}$ II regions. Most important, the slopes are not the same for the three clouds. A fit of $N \propto r^{\gamma}$ over the whole range of column density and radii (starting at the resolution limit and ignoring a possible flattening toward the cloud center) gives values of $\gamma=-0.5 \pm 0.08,-0.8 \pm 0.1$, and $-1.5 \pm 0.15$ for Auriga, Carina, and NGC 3603. The steeper slope of NGC 3603 already becomes obvious purely by visual inspection. Assuming a centrally condensed, spherical density distribution, this corresonds to density profiles $\rho(r) \sim N r^{-1} \sim r^{\gamma-1} \sim r^{-\alpha}$ with $\alpha=1.5,1.8$, and 2.5. These values correspond reasonably well to the ones we also derived from fitting the power-law tail of the PDF (1.78, $1.75,2.5$ ) where we assumed an equivalent density profile (see the studies of the link between PDF and self-gravity by Klessen 2000; Kritsuk et al. 2011; Federrath \& Klessen 2013; Girichidis et al. 2014). In any case, we do not expect a one-to-one correspondence because we do not sample exactly the same areas.

The collapse of an isothermal sphere has been studied for a long time (Larson 1969; Penston 1969; Shu 1977; Whitworth \& Summers 1985), and though all models start with different initial conditions, they arrive at the same $\alpha=2$ for early stages and $\alpha=1.5$ after a singularity formed at the center of the sphere. Our slope determinations from the column density profiles and the power-law tails now clearly point toward a scenario in which gravitational collapse is the dominating 
N. Schneider et al.: Understanding star formation in molecular clouds. I.
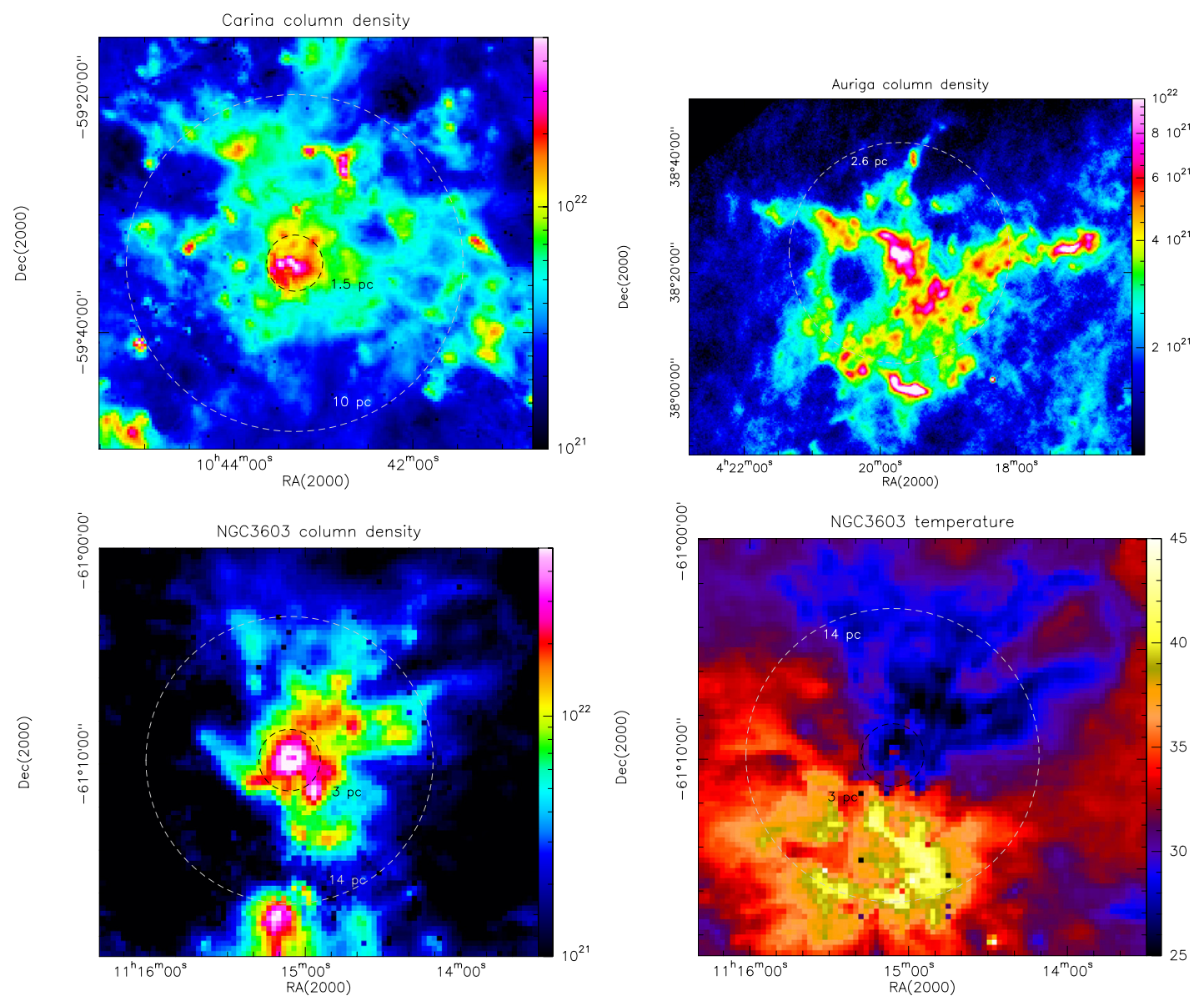

Fig. 12. Zoom into the column density maps of NGC 3603, Carina, and Auriga where we determined the column density profiles shown in Fig. 13. Lower right panel: a temperature map of NGC 3603. The white, dashed circle includes all the pixels we included for the profile and the black, dashed circles for NGC 3603 and Carina outline the area where we observe a possible slope change in the column density profile.

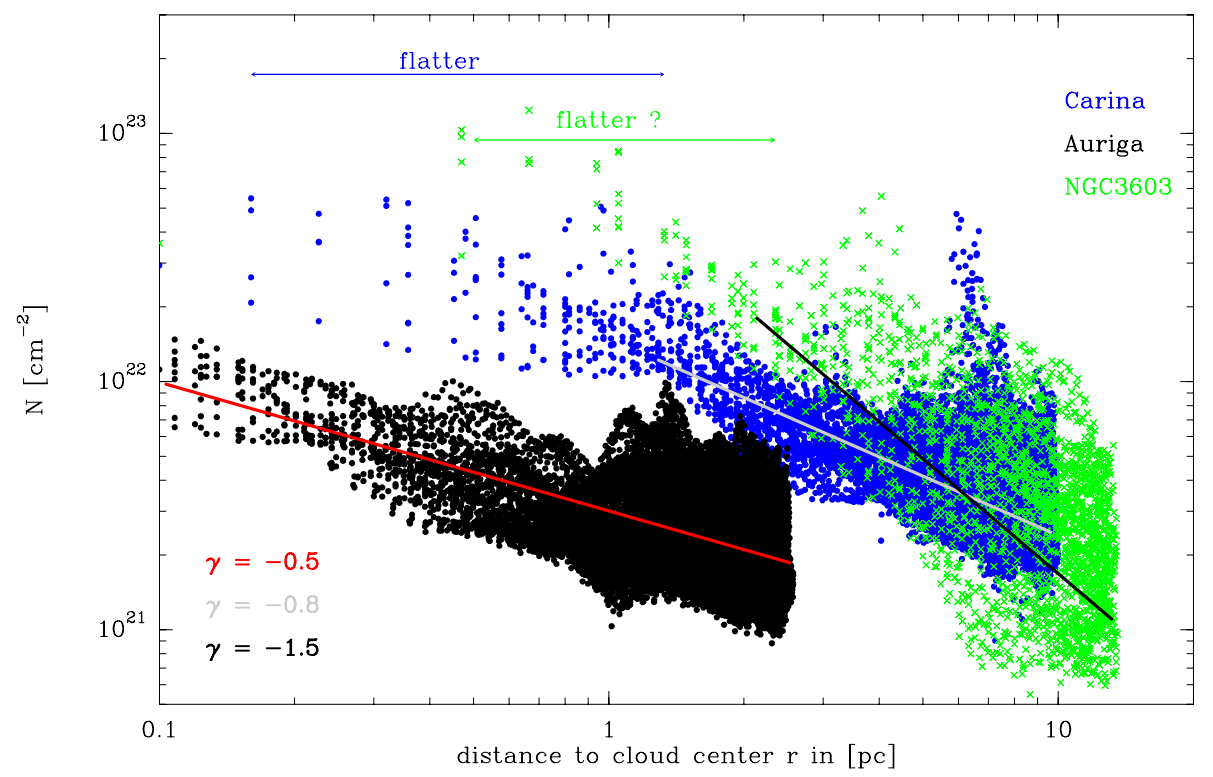

Fig. 13. Column density profiles for NGC 3603, Carina, and Auriga. The angular resolution is $0.03,0.16$, and 0.47 pc for Auriga, Carina, and NGC 3603, respectively. The result of linear regression fits are plotted as a red (Auriga), gray (Carina), and black (NGC 3603) line, the slopes are given in the panel (the error on the slopes is $\sim 0.1$ ).

physical process. We cannot judge whether the cloud as a whole is collapsing or only the individual clumps, cores, and filaments inside the cloud. However, from a theoretical point of view, this does not make any difference. Girichidis et al. (2014) state that "If each density parcel collapses on its free-fall timescale then the evolution of the overall PDF is independent of the number of fragements and the details of the fragmentation process". 
Another argument for the importance of self-gravity comes from the Jeans instability. The number of Jeans masses ${ }^{8} N_{\mathrm{J}}=$ $M / M_{\mathrm{J}}$ of our clouds in the sample is 2040 for NGC 3603, 1350 for Carina, 1100 for Maddalena, and 110 for Auriga. Even under the simplified assumption of uniform density, which gives a lower limit on the number of Jeans masses, all clouds are orders of magnitude more massive than the average Jeans mass. Moreover, from the structural analysis we see that all of the objects are not at uniform density, but are highly structured. The Jeans mass in the regions of interest thus drops, which gives even higher numbers. For a density contrast of 100 , which is certainly not an unrealistic assumption, the above values increase by an order of magnitude, implying that we definitely should expect signs of self-gravity.

It should be noted that for NGC 3603, we find an $\alpha$ that is larger than two that does not correspond to free-fall alone. A possible explanation is radiative feedback. Tremblin et al. (2014) showed that the power-law tail of the PDF becomes flatter going from the cloud center toward the interaction zone between an HII region and the cloud. This implies that compression of gas takes place and that self-gravity then takes over in the densest regions to form cores and finally stars. The flat slope of the PDF for NGC 3603 is thus probably also a consequence of radiative feedback. In Fig. 12 we show a temperature map of NGC 3603 where the $\mathrm{H}_{\text {II }}$ region becomes apparent as a high temperature region southeast of the densest and coldest parts of the molecular cloud.

Coming back to the question of whether all molecular cloud types have the same column density structure, the answer seems to be no. The structure is the same at low and moderate column densities up to the start of the PDF tail, but from the column density profiles and the flatter power-law tails in the PDF seen for the massive SF regions NGC 3603 and Carina, we find an assembly of denser gas in smaller cloud volumes compared to low-mass SF regions. Studying the distribution of mass at different $A_{v}$ scales (see next section) illustrates this behavior.

\subsection{Distribution of masses}

Figures 14 and 15 show the fraction of mass in percent at different $A_{v}$-levels, i.e. column density thresholds (in log-log space and log-lin space to be consistent with Lada et al. 2010; and Kainulainen et al. 2013). In general, the cumulative mass fraction is dominated by the column densities (masses) making up the power-law tail of the PDF. Figure 14 shows that the curves follow a power-law with an exponent of $s+1$, resulting from the direct integration of the PDF to obtain the cumulative mass function, until a fall-off where the PDF becomes noisy due to a lower statistics (which is also true for the cumulative mass functions, see Figs. 14 and 15). In principle the cumulative mass function is derived from the PDF as

$$
\begin{aligned}
M\left(A_{V}\right) \propto \int_{0}^{A_{V}} \frac{\mathrm{d} N\left(A_{V}^{\prime}\right)}{\mathrm{d} A_{V}} A_{V}^{\prime} \mathrm{d} A_{V}^{\prime} & =\int_{0}^{A_{V}} \frac{\mathrm{d} N}{\mathrm{~d} \eta} \frac{\mathrm{d} \eta}{\mathrm{d} A_{V}} A_{V}^{\prime} \mathrm{d} A_{V}^{\prime} \\
& =\int_{0}^{A_{V}} \frac{\mathrm{d} N}{\mathrm{~d} \eta} \frac{A_{V}^{\prime}}{A_{V}^{\prime}} \mathrm{d} A_{V}^{\prime}=\int_{0}^{A_{V}} \frac{\mathrm{d} N}{\mathrm{~d} \eta} \mathrm{d} A_{V}^{\prime} .
\end{aligned}
$$

For any power-law range of the PDF with exponent $s$, we thus expect the integral to have the corresponding slope $s+1$.

\footnotetext{
$8 M_{\mathrm{J}} \propto T^{3 / 2} \rho^{-1 / 2}$ with temperature $T=20 \mathrm{~K}$ as a typical average value, and density $\rho=M / V$, assuming uniform density, and volume $V=(M / \Sigma)^{3 / 2}$ with the mass $M$ and surface density $\Sigma$ of the cloud from Table 1.
}

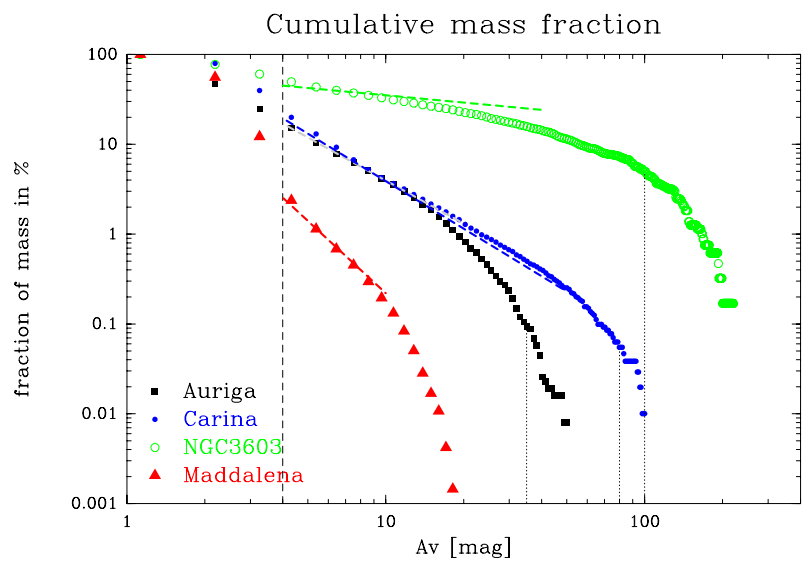

Fig. 14. Log-log display of the mass fraction in percent above the $A_{v}$-level indicated in the $x$-axis for the four clouds in our sample. The curves with the respective slopes from the PDF are overplotted as dashed lines. The short dotted vertical lines indicate the conservative $A_{v}$-level above the pixel number becomes low and thus less reliable. The long dashed line outlines $A_{v}=4$.

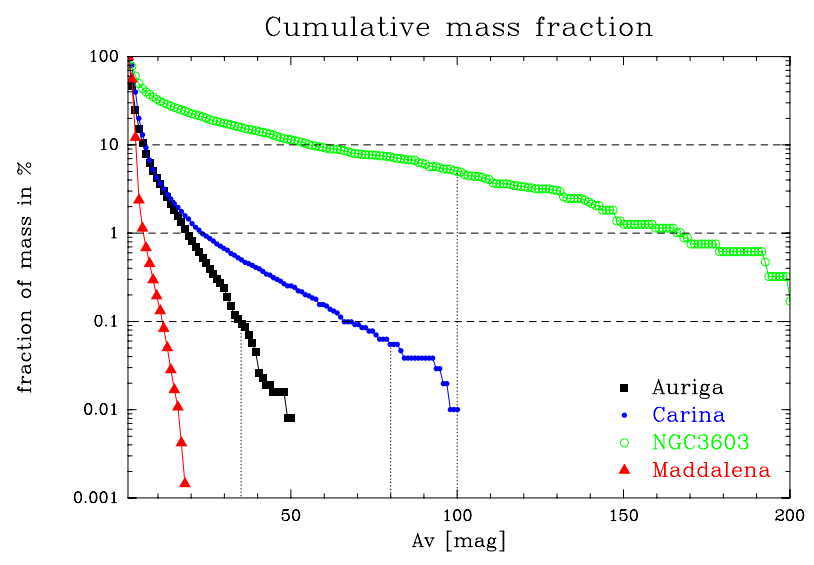

Fig. 15. Log-lin display of the mass fraction in percent above the $A_{v}$-level indicated in the $x$-axis for the four clouds in our sample. The short dotted vertical lines indicate the conservative $A_{v}$-level above the pixel number becomes low and thus less reliable.

The main difference between the cumulative mass functions therefore stems from the different slopes $s$ of the PDF tails. NGC 3603 with the lowest exponent shows a shallow mass function providing a large fraction of high-mass contributions. However, the correspondence here with the slope of the powerlaw tail slope of the PDF is not as good, probably because we left out a larger fraction of high column densities. Comparing this result with the models of Federrath \& Klessen (2013) that were used in Kainulainen et al. (2013), it becomes obvious that NGC 3603 fits very well into the scenario where the higher fraction of dense gas is due to compressive driving. In the extreme case of simulations with only compressive modes, the flat slopes are even nearly independent of the SFE. In the case of mixed driving, magnetic fields have a small impact but more importantly, a higher SFE flattens the slope. Without going into a detailed comparison to these models, which will be done in a forthcoming paper, we conclude that external compression plays the most important role in shaping the column density structure of NGC 3603, consistent with what we deduced from the PDF and column density profile study.

It is interesting to compare the behavior of Carina and Auriga, both having about the same slope for the power-law tail 
of the PDF but a different column density range of the powerlaw fit. Consequently, the cumulative mass functions of both maps have the same slope over the common mass range, but the low-mass star-forming regions break off at lower masses/column densities. In a linear mass scaling, the break-off behavior, i.e. contributions around the upper column density where the powerlaw tail ends, appear more prominent. They represent the few densest cores that might be the places of high-mass or low-mass star formation.

\section{Conclusions}

In this paper, we present column density maps of four molecular clouds obtained from Herschel. These are the Auriga and Maddalena clouds, forming low-mass stars, and Carina and NGC 3603 that are UV-illuminated, high-mass star-forming GMCs. We present a simple method of correcting for LOS contamination from fore- or background clouds by removing a constant layer of emission and then study the effect of this procedure on the resulting column density structure and the PDFs of column density in observations and simulations. Our findings are:

- The PDFs for all observed clouds become broader, the peak shifts to lower column densities, and the power-law tail of the PDF for higher column densities flattens after correction.

- We simulated the effect of LOS contamination by generating a PDF with typical observational parameters, consisting of a lognormal part and a power-law tail, and then "contaminated" this PDF by adding a constant level to all map values. The simulations show that LOS-contamination strongly compresses the lognormal part of the PDF, consistent with what is observed for distant clouds. The peak of the PDF and the value where the PDF turns from lognormal into a powerlaw tail $\left(A_{v}(\mathrm{DP})\right)$ increases, and the slope of the power-law tail becomes steeper.

- We created plots in which for various contamination levels the change in the PDF width $\left(\sigma_{\eta}\right)$ and slope of the power-law tail $(s)$ can be assessed. For a contamination of $\Delta A_{v}=2 \mathrm{mag}$, $\sigma_{\eta}$ is already reduced by more than a factor two, and $s$ has steepened from -2.0 to -2.4 .

- The convolution of the map with the beam has only a minor effect on the high-column density tail of the PDF and no influence on the lower density lognormal distribution. Resolution effects are thus less important when analyzing column density PDFs and cumulative mass functions.

- All observed PDFs that were corrected for LOScontamination have a lognormal part for low column densities with a peak at $A_{v} \sim 2$ mag and a deviation point (DP) from the lognormal at $A_{v}$ (DP) $\sim 4-5 \mathrm{mag}$. For higher column densities, all PDFs have a power-law tail with an average slope of $-2.6 \pm 0.5$.

- Assuming an equivalent spherical density distribution $\rho \propto$ $r^{-\alpha}$, this average slope corresponds to an exponent $\alpha_{\mathrm{PDF}}=$ $1.9 \pm 0.3$ consistent with the view that the gas in the powerlaw tail is dominated by self-gravity (local free-fall of individual cores and global collapse of gas on larger scales, such as filaments).

- Our PDF study suggests that there is a common column density break at $A_{v} \sim 4-5$ mag for all cloud types where the transition between supersonic turbulence and self-gravity takes place. Our value is lower than the one found by Froebrich \& Rowles (2010) with $A_{v}=6.0 \pm 1.5 \mathrm{mag}$ but consistent with cloud simulations of self-gravitating turbulent gas (Ward et al. 2014).
- The average column density for low-mass SF regions $(1.8 \times$ $10^{21} \mathrm{~cm}^{-2}$ ) is slightly lower than the one for high-mass SF clouds $\left(3.0 \times 10^{21} \mathrm{~cm}^{-2}\right)$. Because of the small sample (four clouds), and the uncertainties introduced by cropping effects, it is not clear whether this difference is statistically significant.

- Radial column density profiles of three clouds in our sample show a distribution that is compatible with (global) gravitational collapse for Auriga and Carina (slopes correspond to $\alpha=1.5$ and 1.8), but requires an additional compression process for NGC 3603 ( $\alpha=2.5)$. We suggest that compression from the expanding ionization fronts from the associated $\mathrm{H}$ II region leads to a forced collapse. This is consistent with the higher mass fraction at higher column densities in the cumulative mass function and corresponds well with numerical simulations based on compressive driving.

- In view of the differences observed for the slopes of the power-law tail of the PDF and the variation in the run of the column density profiles, we find that the column density structure of clouds forming low-mass stars and the ones forming massive stars are not the same.

- The cumulative mass distributions for high-mass SF regions is shallower than the one for low-mass SF clouds for high column densities, indicating a higher concentration of dense gas in smaller cloud volumes.

Acknowledgements. N.S., S.B., and P.A. acknowledge support by the ANR11-BS56-010 project "STARFICH". V.O., N.S., P.G., and R.S.K. acknowledge subsidies from the Deutsche Forschungsgemeinschaft, priority program 1573 ("Physics of the Interstellar Medium"). R.S.K. acknowledges support by the collaborative research project SFB 881 ("The Milky Way System", subprojects B1, B2, and B5), and support from the European Research Council under the European Communities Seventh Framework Program (FP7/20072013) via the ERC Advanced Grant STARLIGHT (project number 339177). C.F. acknowledges funding provided by the Australian Research Council's Discovery Projects (grants DP130102078 and DP150104329). T.Cs. acknowledges financial support from the ERC Advanced Grant GLOSTAR under contract No. 247078. Ph. André acknowledges financial support from the ERC Advanced Grant ORISTARS under contract No. 291294. V.O. and N.S. acknowledge support by the Deutsche Forschungsgemeinschaft, DFG, through project number OS 177/2-1. We thank an anonymous referee for the detailed comments that improved the paper.

\section{References}

Alves, J., Lombardi, M., \& Lada, C. J. 2014, A\&A, 565, A18 Alves de Oliveira, C., Schneider, N., Merin, B., et al. 2014, A\&A, 568, A98 André, Ph., Men'shchikov, A., Bontemps, S., et al. 2010, A\&A, 518, L102

André, Ph., Di Francesco, J., Ward-Thompson, D., et al. 2014, Protostars and Planets VI (University of Arizona Press), eds. H. Beuther, R. S. Klessen, C. Dullemond, \& Th. Henning [arXiv: 1312.6232]

Arzoumanian, D., André, Ph., Didelon, P., et al. 2011, A\&A, 529, L1

Ballesteros-Paredes, J., \& Mac Low, M.-M. 2002, ApJ, 570, A734

Ballesteros-Paredes, J., Vázquez-Semadeni, E., \& Gazol, A. 2011, MNRAS, 416, 1436

Bohlin, R. C., Savage, B. D., \& Drake, J. F. 1978, ApJ, 224, A132

Brunt, C. M., Federrath, C., \& Price, D. J. 2010, MNRAS, 405, L56

Burkhart, B., Ossenkopf, V., Lazarian, A., \& Stutzki, J. 2013, ApJ, 771, A122

Cambrésy, L., Rho, J., Marshall, D. J., et al. 2011, A\&A, 527, A141

Cambrésy, L., Marton, G., Feher, O., et al. 2013, A\&A, 557, A29

Carlhoff, P., Schilke, P., Nguyen-Luong, Q., et al. 2013, A\&A, 560, A24

Cho W., \& Kim J. 2011, MNRAS, 410, L8

Federrath, C., \& Klessen, R. S. 2013, ApJ, 763, A51

Federrath, C., \& Klessen, R. S. 2012, ApJ, 761, A156

Federrath, C., Roman-Duval, J., Klessen, R. S., et al. 2010, A\&A, 512, A81

Federrath, C., Glover, S., Klessen, R. S., \& Schmidt, W. 2008, Phys. Scr. 132, 4025

Fischera, J., \& Dopita, M. A. 2004, ApJ, 611, 919

Froebrich, D., \& Rowles, J. 2010, MNRAS, 406, 1350

Gaczkowski, B., Preibisch, T., Ratzka, T., et al. 2012, A\&A, 549, A67

Girichidis, P., Konstandin, L., Whitworth, A. P., Klessen, R. S., et al. 2014, ApJ, 781,91 
Goldsmith, P. F., Heyer, M., Narayanan, G., et al. 2008, ApJ, 680, 428 Goodman, A. A., Pineda, J., \& Schnee, S. L. 2009, ApJ, 692, 91 Griffin, M., Abergel, A., Abreau, A., et al. 2010, A\&A, 518, L3

Hacar, A., Tafalla, M., Kauffmann, J., \& Kovacs, A. 2013, A\&A, 554, A55

Harvey, P., Fallscheer, C., Ginsburg, A., et al. 2013, ApJ, 764, 133

Hennebelle, P., \& Chabrier, G. 2008, ApJ, 684, 395

Hennebelle, P., \& Chabrier, G. 2009, ApJ, 702, 1428

Hennemann, M., Motte, F., Schneider, N., et al. 2012, A\&A, 543, L3

Heyer, M. H., Krawczyk, C., \& Duval, J. 2009, ApJ, 699, 1092

Hill, T., Motte, F., Didelon, P., et al. 2011, A\&A, 533, A94

Kainulainen, J., Beuther, H., Henning, T., \& Plume, R. 2009, A\&A, 508, L35

Kainulainen, J., Beuther, H., Banerjee, R., et al. 2011, A\&A, 530, A64

Kainulainen, J., Federrath, C., \& Henning, T. 2013, A\&A, 553, L8

Klessen, R. S. 2000, ApJ, 535, 869

Kritsuk, A. G., Norman, M. L., \& Wagner, R. 2011, ApJ, 727, L20

Krumholz, M., \& McKee, C. F. 2005, ApJ, 630, 250

Lada, C. J., Lada, E. A., Clemens, D. P., \& Bally, J. 1994, ApJ, 429, 694

Lada, C. J., Lombardi, M., \& Alves, J. 2009, ApJ, 703, 52

Lada, C. J., Lombardi, M., \& Alves, J. 2010, ApJ, 724, 687

Larson, R. B. 1969, MNRAS, 145, 271

Larson, R. B. 1981, MNRAS, 194, 809

Lombardi, M. 2009, A\&A, 493, 735

Lombardi, M., \& Alves, J. 2001, A\&A, 377, 1023

Lombardi, M., Alves,J., \& Lada, C. J. 2006, A\&A, 454, 781

Lombardi, M., Lada, C. J., \& Alves, J. 2008, A\&A, 489, 143

Lombardi, M., Alves, J., \& Lada, C. J. 2010, A\&A, 519, L7

Molina, F. Z., Glover, S., Federrath, C., \& Klessen, R. S. 2012, MNRAS, 423, 2680

Molinari, S., Swinyard, B., Bally, J. et al. 2010, A\&A, 518, L100

Motte, F., Ngyuen-Luong, Q., Schneider, N., et al. 2014, A\&A, 571, A32

Padoan, P., \& Nordlund, A. A. 2002, ApJ 576, 870
Padoan, P., Jones, J. T. \& Nordlund, A. A. 1997, ApJ, 474, 730 Palmeirim, P., André, Ph., Kirk, J., et al. 2013, A\&A, 550, A38 Passot, T., \& Vazquez-Semadeni, E. 1998, Phys. Rev. E, 58, 4 Penston, M. V. 1969, MNRAS, 144, 425

Pilbratt, G., Riedinger, J., Passvogel, T., et al. 2010, A\&A, 518, L1

Poglitsch, A., Waelkens, C., Geis, N., et al. 2010, A\&A, 518, L2

Polychroni, D., Schisano, E., Elia, D., et al. 2013, ApJ, 777, L33

Preibisch, T., Roccatagliata, V., Gaczkowski, B., \& Ratzka, T. 2012, A\&A, 541, A132

Rivera-Ingraham, A., Martin, P. G., Polychroni, D., et al. 2013, ApJ, 766,85

Roccatagliata, V., Preibisch, T., Ratzka, T., \& Gaczkowski, B. 2013, A\&A, 554, A6

Roussel, H. 2013, PASP, 125, 1126

Rowles, J., \& Froebrich, D. 2009, MNRAS, 395, 1640

Roy, A., André, Ph., Palmeirim, P., et al. 2014, A\&A, 562, A38

Russeil, D., Schneider, N., Anderson, L., et al. 2013, A\&A, 554, A42

Schneider, N., \& Brooks, K. 2004, PASA, 21, 290

Schneider, N., Csengeri, T., Bontemps, S., et al. 2010, A\&A, 520, A49

Schneider, N., Bontemps, S., Simon, R., et al. 2011, A\&A, 529, A1

Schneider, N., Csengeri, T., Hennemann, M., et al. 2012, A\&A, 540, L11

Schneider, N., André, Ph., Könyves, V., et al. 2013, ApJ, 766, L17

Schneider, N., Csengeri, T., \& Klessen, R. 2014, A\&A, submitted [arXiv: 1406.3134]

Shu, F. H. 1977, ApJ, 214, 488

Stutzki, J., Bensch, F., Heithausen, A., Ossenkopf, V., \& Zielinsky, M. 1998, A\&A, 336, 697

Tremblin, P., Schneider, N., Minier, V., et al. 2014, A\&A, 564, A106

Vázquez-Semadeni, E., \& Garcia, N. 2001, ApJ, 557, 727

Ward, R. L., Wadsley, J., \& Sills, A. 2014, MNRAS, 445, 1575

Whitworth, A., \& Summers, D. 1985, MNRAS, 214, 1 
N. Schneider et al.: Understanding star formation in molecular clouds. I.
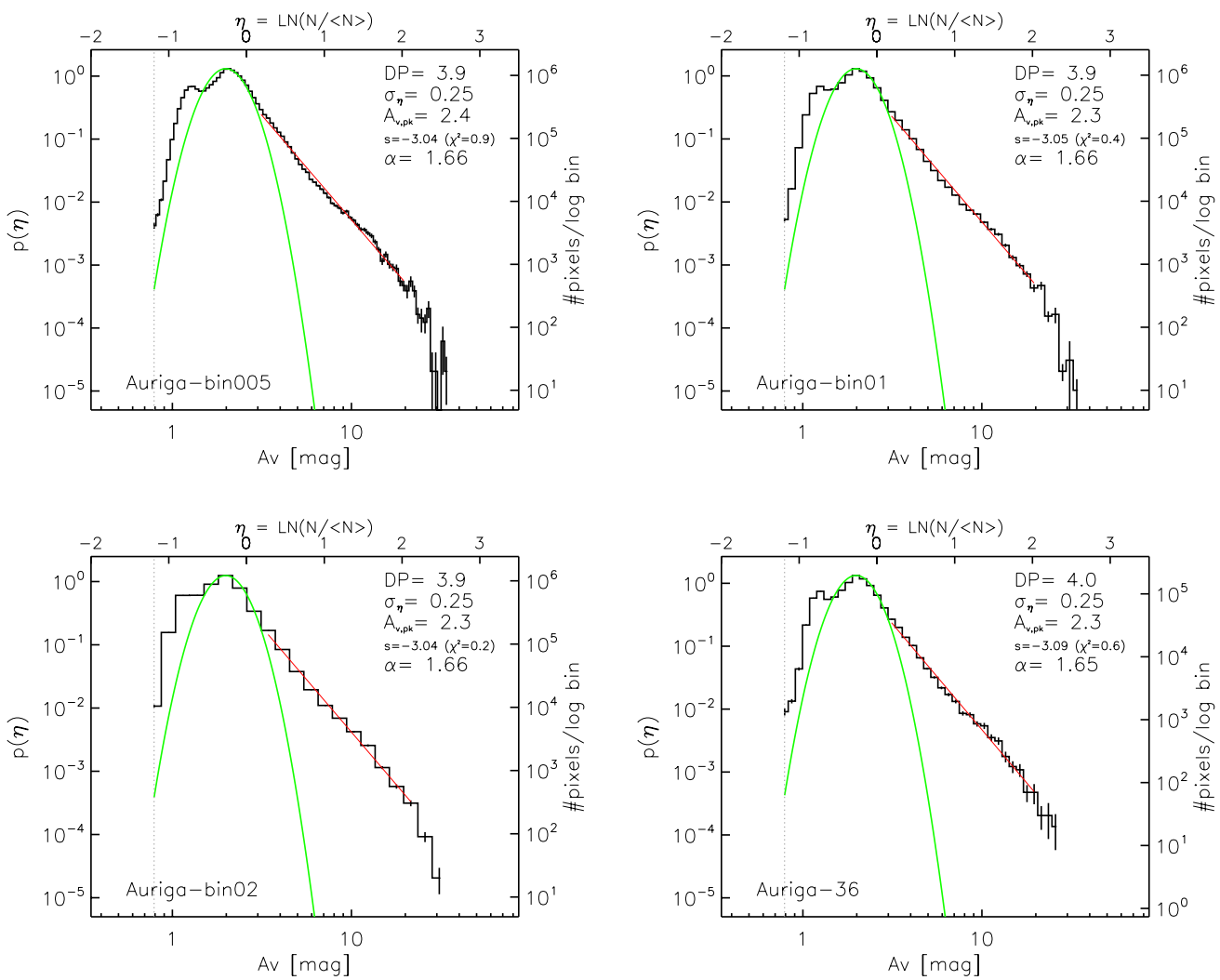

Fig. A.1. PDFs obtained from the orginal (not LOS-corrected) Auriga column density map with different bin sizes (0.05, 0.1, and 0.2) and a PDF with a binsize of 0.1 but on a grid of 36" (lower, right panel). The vertical dashed line indicates the noise level of the map. The left $y$-axis gives the normalized probability $p(\eta)$, the right $y$-axis the number of pixels per log bin. The upper $x$-axis is the visual extinction and the lower $x$-axis the logarithm of the normalized column density. The green curve indicates the fitted PDF (we fixed the width of the PDF in order to avoid the low column density component being included). The red line indicates a power-law (linear regression) fit to the high $A_{v}$ tail (the start- and end-point were fixed to the same values for each PDF). Inside each panel, we give the value where the PDF peaks $\left(A_{\mathrm{pk}}\right)$, the deviation point from lognormal to power-law tail (DP), the dispersion of the fitted PDF $\left(\sigma_{\eta}\right)$, the slope $s$ and the $X^{2}$ of the fit, and the exponent $\alpha$ of an equivalent spherical density distribution.

\section{Appendix A: Effects of binning and cropping on the PDF}

Figure A.1 shows the effect on the PDF of varying the binsize. We started first from the original column density map on a $14^{\prime \prime}$ grid, which approximately corresponds to Nyquist sampling for an angular resolution of the data of $36^{\prime \prime}$. It becomes obvious that there is basically no effect on the derived values of $A_{v}$ (peak), $A_{v}$ (DP), and slope. For the fit, we fixed only the width of the PDF in order to avoid the lower column density peak, arising from a seperate component, being taken into account. A binsize of 0.1 turns out to be the best choice for our data because finer sampling (0.05) increases the statistical noise for the higher column density range, and a lower sampling (0.2) smoothes out features in the PDF that can still be significant.

In Fig. A.2 we investigate the effect of "field-cropping", which is how the PDF shape changes when only pixels above a certain threshold are considered, using the Auriga cloud as an example. First, we observe that for high column density ranges, i.e. above the peak of the lognormal distribution, the PDF is only represented by a power-law tail all with the same slope, independent of $A_{v}$-threshold. Small variations in the PDF shape are due to the normalization process. (The average column density changes for each threshold-value). As a consequence, PDFs obtained by "cropping" images at high column densities, such as PDFs for IRDCs in IR-quiet molecular clouds, should thus be strongly dominated by the power-law tail. This was recently confirmed observationally by Schneider et al. (2014). Toward the low column density range, we constructed PDFs above threshold values of $A_{v}=0.2$ (noise level), $A_{v}=0.5$, and $A_{v}=0.8$ (background level). We do not observe a significant effect on the PDF shape when we change the threshold, though it is clear that for the lowest column densities, the clouds in our Herschel maps are not completely sampled. 

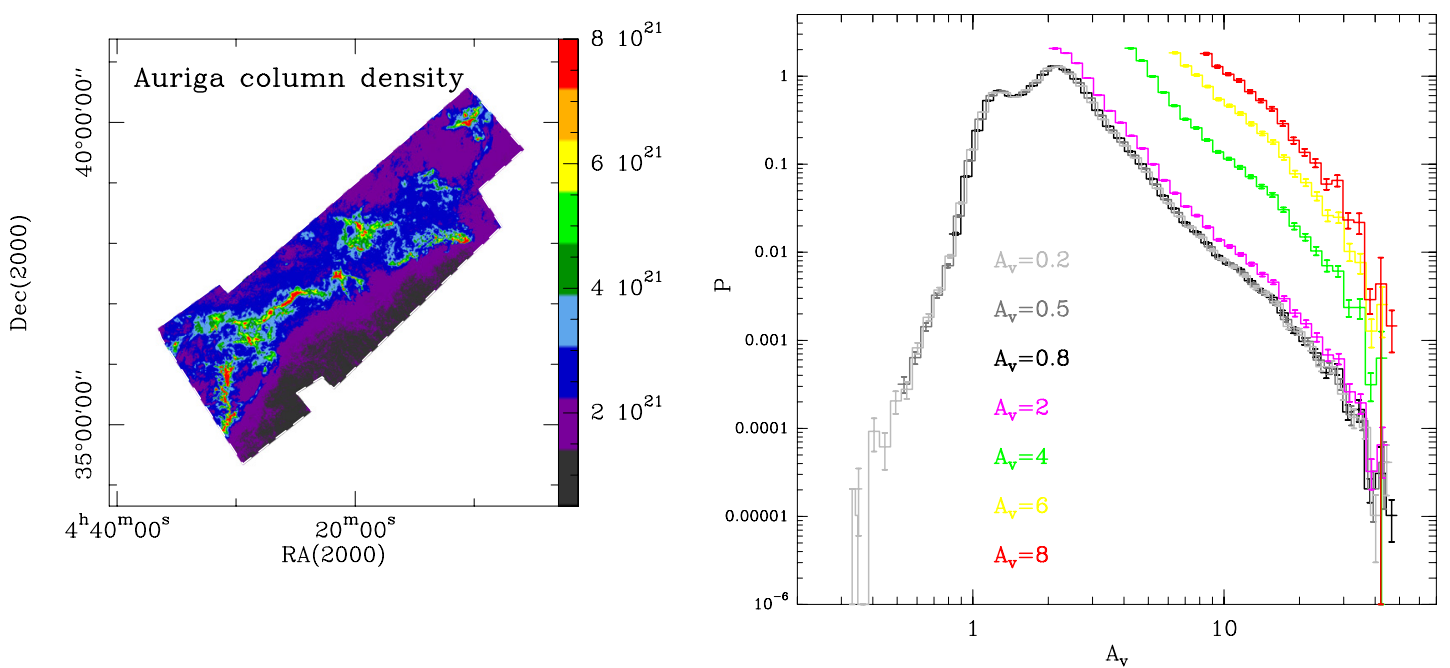

Fig. A.2. Left: column density map of Auriga (without correction for LOS-contamination), showing the emission distribution at various thresholds of $A_{v}$. The colors correspond to the PDFs (right panel) constructed above $A_{v}$ levels of $0.8,2,4,6$, and 8 . The $y$-axis gives the normalized probability $p(\eta)$, and the $x$-axis the visual extinction.
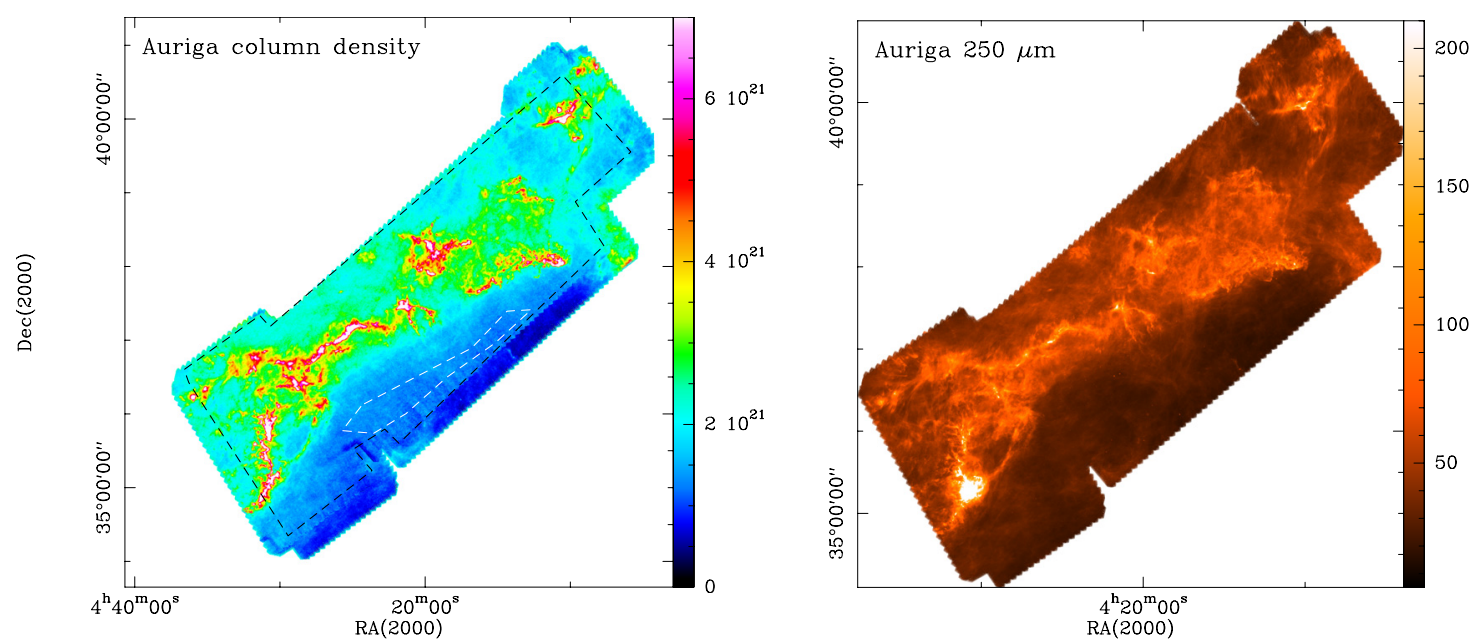

Fig. B.1. Left: Herschel column density map of the Auriga cloud in $\left[\mathrm{cm}^{-2}\right]$. The black dashed polygon indicates the pixels from which the PDF was determined. This is the common overlap region of SPIRE and PACS in which the column density map was determined from the SED fit using the 4 wavelengths. Outside this polygon, the fit relies only on SPIRE and it less reliable. The white polygon outlines the area used for determining the background/foreground level of contamination of the map. Right: SPIRE $250 \mu \mathrm{m}$ map in units [MJy/sr].

\section{Appendix B: Herschel maps of molecular clouds}

All maps presented in this paper - and in the furthcoming ones - were treated in the same way with regard to data reduction and determination of the column density maps. For the data reduction of the Herschel wavelengths, we used the HIPE10 pipeline, including the destriper task for SPIRE (250, 350, $500 \mu \mathrm{m})$, and HIPE10 and scanamorphos v12 (Roussel et al. 2013) for PACS (70" and 160", Poglitsch et al. 2010). The SPIRE (Griffin et al. 2010) maps include the turnaround-data (when the satellite changed mapping direction for the scan) and were calibrated for extended emission.

The procedure for making column density and temperature maps is independent of the data reduction process and follows the scheme described in Schneider et al. (2012). The column density maps were determined from a pixel-to-pixel graybody fit to the red wavelength of PACS $(160 \mu \mathrm{m})$ and SPIRE $(250,350$, $500 \mu \mathrm{m}$ ). We did not include the $70 \mu \mathrm{m}$ data because we focus on the cold dust and not UV-heated warm dust (see Sect. 4.1 in
Russeil et al. 2013 for more details on this issue). The maps were first convolved to have the same angular resolution of $36^{\prime \prime}$ and then regridded on a $14^{\prime \prime}$ raster. All maps have an absolute flux calibration, using the ZEROPOINTCORRECTION task in HIPE10 for SPIRE and IRAS maps for PACS.

The correction for SPIRE works in such a way that that a cross-calibration with Planck maps at 500 and $350 \mu \mathrm{m}$ is performed. We emphasize that such a correction is indispensable for accurately determining column density maps. For the SED fit, we fixed the specific dust opacity per unit mass (dust+gas) approximated by the power law $\kappa_{v}=0.1(v / 1000 \mathrm{GHz})^{\beta} \mathrm{cm}^{2} / \mathrm{g}$ and $\beta=2$ and left the dust temperature and column density as free parameters. We checked the SED fit of each pixel and determined from the fitted surface density the $\mathrm{H}_{2}$ column density. For the transformation $\mathrm{H}_{2}$ column density into visual extinction, we used the conversion formula $N\left(\mathrm{H}_{2}\right) / A_{v}=0.94 \times 10^{21} \mathrm{~cm}^{-2} \mathrm{mag}^{-1}$ (Bohlin et al. 1978). The angular resolution of the column density maps is $\sim 36^{\prime \prime}$, and they are shown in Figs. B.1 to B.4, together with SPIRE $250 \mu \mathrm{m}$ 
N. Schneider et al.: Understanding star formation in molecular clouds. I.
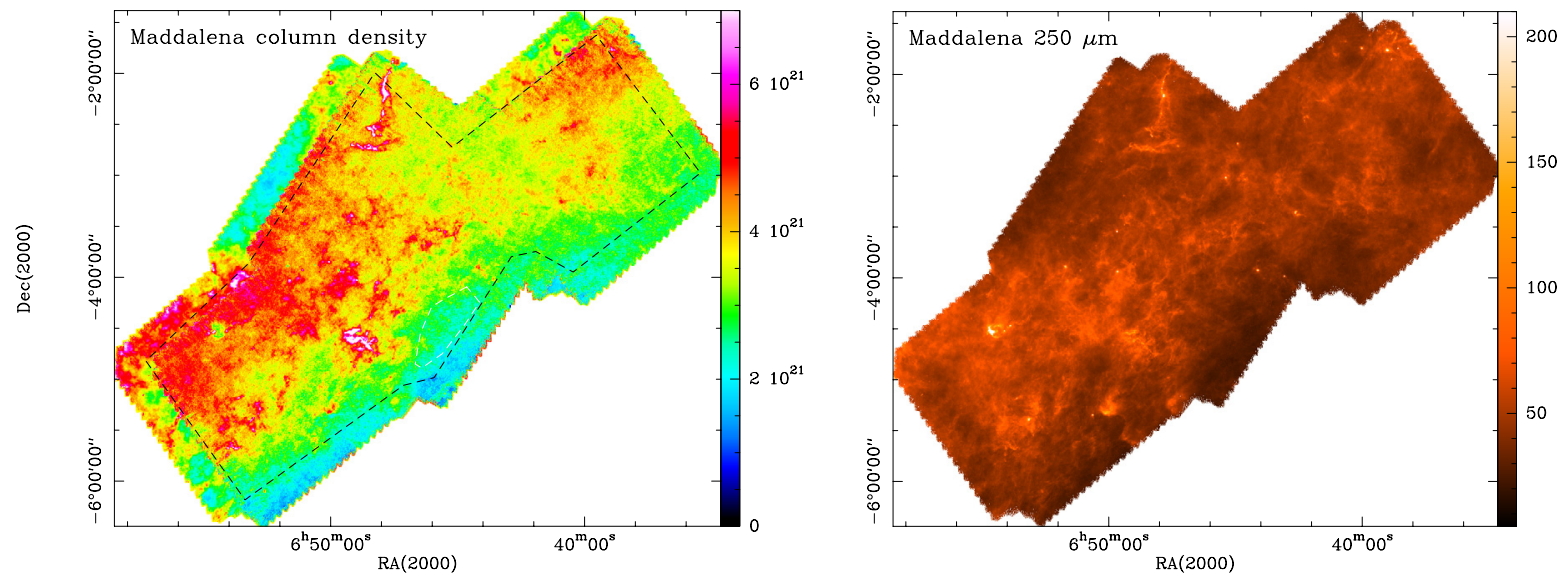

Fig. B.2. Left: Herschel column density map of the Maddalena cloud in $\left[\mathrm{cm}^{-2}\right]$. All other parameters as in Fig. B.1. Right: SPIRE $250 \mu \mathrm{m}$ map in units $[\mathrm{MJy} / \mathrm{sr}]$.
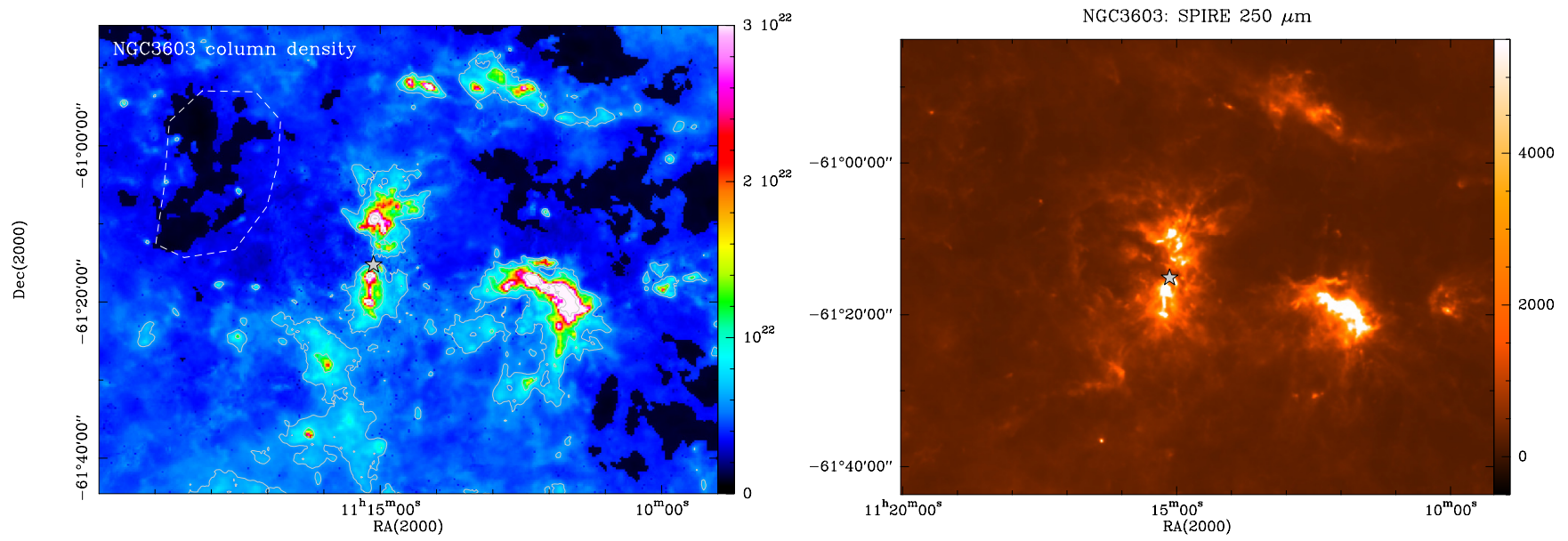

Fig. B.3. Left: Herschel column density map of NGC 3603 in $\left[\mathrm{cm}^{-2}\right]$. The contour levels are 3, 6, 10, and $20 \times 10^{21} \mathrm{~cm}^{-2}$. All other parameters as in Fig. B.1. Right: SPIRE $250 \mu \mathrm{m}$ map in units [MJy/sr]. The gray star indicates the location of the central cluster.
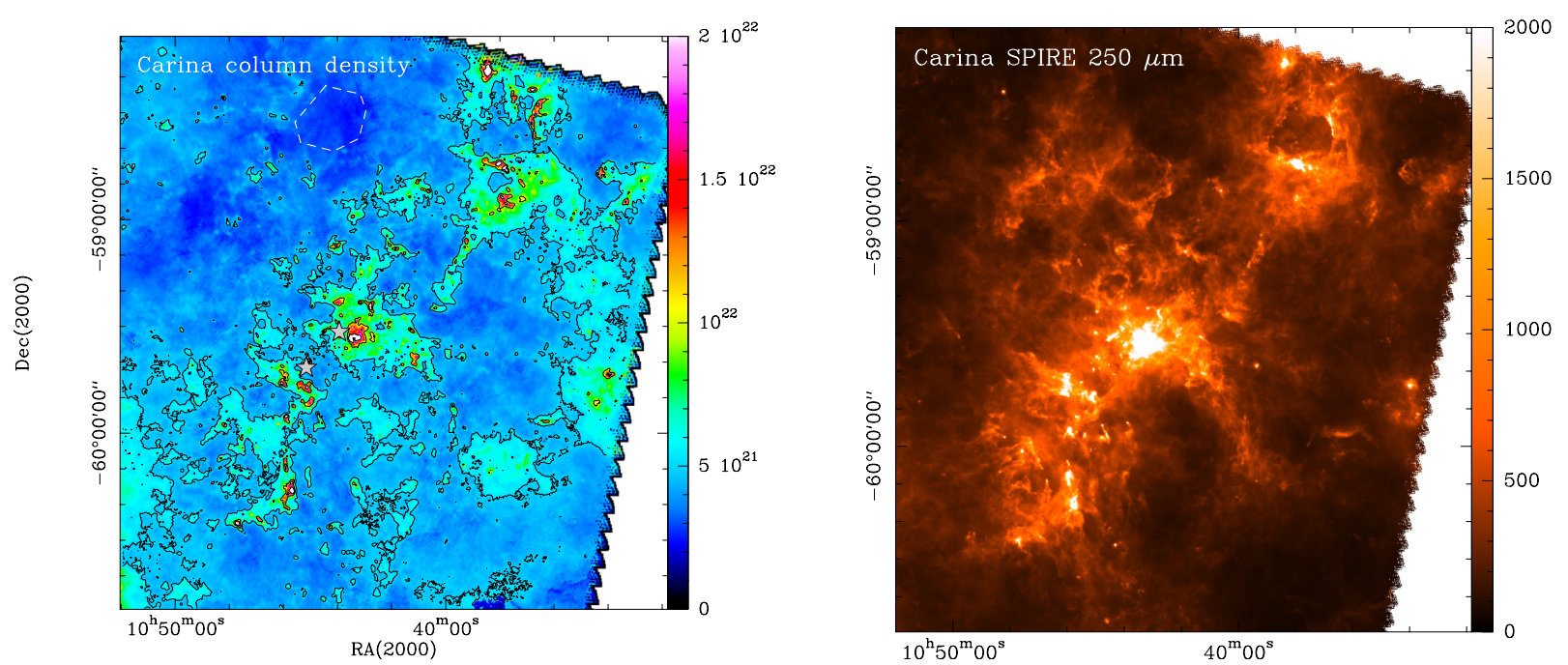

Fig. B.4. Left: Herschel column density map of Carina in $\left[\mathrm{cm}^{-2}\right]$. The contour levels are 5, 10, and $20 \times 10^{21} \mathrm{~cm}^{-2}$. All other parameters as in Fig. B.1. Right: SPIRE $250 \mu \mathrm{m}$ map in units [MJy/sr]. The gray stars indicate the location of the OB clusters $\operatorname{Tr} 14$ and $\operatorname{Tr} 16$.

images. We estimate the final uncertainties in the Herschel column density maps to be around $\sim 30-40 \%$, mainly arising from the uncertainty in the assumed form of the opacity law, and possible temperature gradients along the LOS (see Roy et al. 2014, for details on the dust opacity law and Russeil et al. 2013, for a quantitative discussion on the various error sources). 